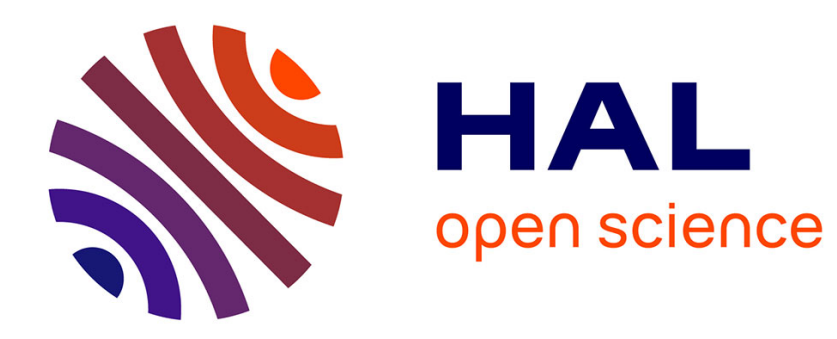

\title{
Pore network model of drying with Kelvin effect
}

Otman Maalal, Marc Prat, Didier Lasseux

\section{To cite this version:}

Otman Maalal, Marc Prat, Didier Lasseux. Pore network model of drying with Kelvin effect. Physics of Fluids, 2021, 33 (2), pp.027103. 10.1063/5.0035651 . hal-03147548

\section{HAL Id: hal-03147548 \\ https://hal.science/hal-03147548}

Submitted on 20 Feb 2021

HAL is a multi-disciplinary open access archive for the deposit and dissemination of scientific research documents, whether they are published or not. The documents may come from teaching and research institutions in France or abroad, or from public or private research centers.
L'archive ouverte pluridisciplinaire HAL, est destinée au dépôt et à la diffusion de documents scientifiques de niveau recherche, publiés ou non, émanant des établissements d'enseignement et de recherche français ou étrangers, des laboratoires publics ou privés. 


\title{
Pore network model of drying with Kelvin effect
}

\author{
Phys. Fluids 33, 027103 (2021); doi: 10.1063/5.0035651
}

\begin{abstract}
Otman Maalal, ${ }^{1,2}$ (D) Marc Prat, ${ }^{1, a)}$ (D) and Didier Lasseux ${ }^{3}$ (D)
AFFILIATIONS

${ }^{7}$ Institut de Mécanique des Fluides de Toulouse (IMFT), Université de Toulouse, CNRS, Toulouse, France

${ }^{2}$ Institut de La Filtration et des Techniques Séparatives (IFTS), Rue Marcel Pagnol, 47510 Foulayronnes, France

${ }^{3}$ I2M, UMR 5295, CNRS, Univ. Bordeaux-Esplanade des Arts et Métiers, 33405 Talence, CEDEX, France

${ }^{a)}$ Author to whom correspondence should be addressed: mprat@imft.fr. Tel.: +33534322883

ABSTRACT

A pore network model of isothermal drying is presented. The model takes into account the capillary effects, the transport of vapor by diffusion, including Knudsen effect, in the gas phase, and the Kelvin effect. The model is seen as a first step toward the simulation of drying in mesoscopic porous materials involving pore sizes between $4 \mathrm{~nm}$ and $50 \mathrm{~nm}$. The major issue addressed with the present model is the computation of the menisci mean curvature radius at the boundary of each liquid cluster in conjunction with the Kelvin effect. The impact of Kelvin effect on the drying process is investigated, varying the relative humidity in the ambient air outside the medium. The simulations indicate that the Kelvin effect has a significant impact on the liquid distribution during drying. The evaporation rate is found to fluctuate due to the menisci curvature variations during drying. The simulations also highlight a noticeable non-local equilibrium effect.
\end{abstract}

\section{INTRODUCTION}

Porous media with pore sizes typically between $2 \mathrm{~nm}$ and $50 \mathrm{~nm}$ are referred to as mesoporous. Mesoporous porous media or partially mesoporous porous media (i.e., with a fraction of the pores in the mesoscopic range) correspond to an important class of materials, such as concrete, clay rocks, ultrafiltration membranes or catalyst, and micro-porous layers of proton exchange membrane (PEM) fuel cells to only cite a few. Evaporation is an important phenomenon to be studied for this class of materials in relation with, for instance, the drying of concrete, ${ }^{1}$ water desalination processes, ${ }^{2}$ electronic cooling, ${ }^{3}$ the transpiration in plants, ${ }^{4}$ or the operation of fuel cells to name only a few. This phenomenon has been the subject of many experimental and modeling studies. As for the capillary (or macroporous) media with pore sizes greater than $50 \mathrm{~nm}$, the drying models for the mesoporous media are generally developed within the framework of the continuum approach to porous media. The main difference between the macroporous and mesoporous materials lies in the significance of the sorption phenomena, ${ }^{5}$ which are taken into account through the consideration of the desorption isotherm in the models. Such a desorption isotherm typically relates the equilibrium liquid saturation in the porous medium and the vapor partial pressure in the surrounding gas. Whereas the corresponding saturations are extremely low with the macroporous media, the desorption isotherm is associated with a significant range of saturations in the case of a mesoporous material. For this reason, the mesoporous materials are also sometimes referred to as hygroscopic, whereas the macroporous materials are referred to as non-hygroscopic or capillary. ${ }^{6}$ Regarding the macroscopic models for mesoporous materials, a distinction can be made between the "static" drying models and the "dynamic" drying models. In a static drying model, only equilibrium saturations are considered as given by the desorption isotherm. This type of model is commonly used for predicting the drying shrinkage of mesoporous materials, e.g., Refs. 7 and 8 and references therein. By contrast, a dynamic (or transient) model aims at predicting the temporal (and spatial) variation of the saturation when the material is exposed to evaporation as well as the temporal variation of the evaporation rate (as well as other variations, mechanical for instance, depending on the considered problem). Typically, in the case of quasi-isothermal drying at the ambient temperature, which is the situation considered in this paper, this type of approach leads to the consideration of a strongly non-linear diffusion equation governing the variation of the moisture content or the saturation, e.g., Ref. 9. Examples of this type of model can be found in Refs. 1, 6, 9, and 10. Contrary to the static models, the dynamic models imply the consideration of the transport phenomena occurring during the drying process. The desorption isotherm is still used to relate the partial vapor pressure and the saturation at the scale of the 
Representative Elementary Volume (REV). The later assumption has been questioned, which leads to the consideration of non-local equilibrium models, ${ }^{11}$ in which the mean vapor pressure in a REV during the dynamic drying process is less than the equilibrium vapor pressure corresponding to the desorption isotherm. Another important issue lies in the transport mechanisms occurring in the pore space during drying. Since these mechanisms are actually lumped into the nonlinear effective diffusion coefficient, it is practically impossible to delineate the various mechanisms at play from the experimental identification of this coefficient. The liquid flow driven by the capillary pressure and the diffusion of the vapor in the gas phase are the most frequently evoked mechanisms. However, the model proposed in Ref. 6 puts a strong emphasis of the impact of the bound water, ${ }^{12}$ i.e., the transport in the very thin liquid films adsorbed on the pore walls. On the other hand, the bound water transport mechanism is not mentioned in Refs. 9 and 10 and most macroscopic models, ${ }^{1}$ where the dominant transport mechanism in the gas occupied region is vapor diffusion.

Insights on the mechanisms at play can be expected from the expanding field of nanofluidics. For instance, some of the experiments reported in Ref. 13 in 20-30 nm slits were consistent with the classical picture of a receding meniscus driven by diffusion (as indicated by the reported classical $L \propto \sqrt{t}$ behavior, where $L$ is the evaporation length, i.e., the distance between the entrance of the slit and the receding meniscus position in the slit). However, the variation of $L$ is found faster that when only pure vapor diffusion is considered. Film flows are then invoked to explain the discrepancy. At first glance, this could be seen as an experimental confirmation of the considerations made in Ref. 6, where both the vapor diffusion transport and the bound water flow are considered as significant transport mechanisms in the pores invaded in the bulk by the gas phase. However, it is known that the slit geometry favors the formation of important corner films ${ }^{14}$ driven by capillarity rather than the disjoining pressure as for the bound water. It is well established that corner films can strongly enhance the evaporation. ${ }^{15,16}$ In other words, it cannot be readily deduced from the experiments reported in Ref. 13 that the bound water transport represents a significant transport mechanism compared to vapor diffusion. Nevertheless, it seems clear that the significance of the transport in thin films must be assessed. Additional interesting information is given from the nanofluidics studies. Under certain circumstances, the drying process is different from the classical receding menisci picture because of the occurrence of cavitation. ${ }^{13,17}$ This leads to a completely different evolution of the phase distribution in the medium. In what follows, the cavitation scenario is not addressed and the classical receding menisci scenario is considered.

The aforementioned short review clearly indicates that the mechanisms at play during the dynamic drying of mesoporous materials are still to be fully clarified and can present important differences compared to the case of macroporous materials. Since the relative significance of the various mechanisms is difficult to assess from the macroscopic models, modeling at the pore scale is considered. In the porous media literature, various techniques are used to simulate transport phenomena at the pore scale. If one disregards the molecular dynamics simulations, which only apply to a few pores over very short time scales, the two main approaches are the direct simulations, e.g., Ref. 18 and the pore network modeling (PNM) approach. In continuity with several previous works, the PNM approach is adopted for the present work. Pore network modeling (PNM) is now a classical technique to study transport phenomena in porous media. The technique has been applied to drying problems since the early 90s (see, e.g., Ref. 19 and references therein). The first model of this type ${ }^{19}$ only took into account the vapor transport by diffusion in the gas phase, together with the capillary effects. Since then, many effects have been included in drying PNM, and this is reviewed in Refs. 20 and 21. However, to the best of our knowledge, all the drying PNM reported so far have been applied to macroporous porous media characterized by pore sizes greater than $50 \mathrm{~nm}$. The objective of this article is, therefore, to contribute to the development of a pore network model for the study of drying in mesoporous materials. As a first step toward the detailed simulation of drying in mesoporous materials, a PNM taking into account the Kelvin effect is presented in what follows. The Kelvin effect refers to the modification of the equilibrium vapor pressure, $P_{\text {vequ. }}$, due to the curvature of liquid-gas interfaces and can be computed from the Kelvin relationship which takes the following expression:

$$
R H_{\text {equ. }}=\frac{P_{\text {vequ. }}}{P_{v s}}=\exp \left(-\frac{M_{v}}{R T} \frac{2 \gamma \cos \theta}{\rho_{\ell} r}\right) .
$$

Here, $P_{v s}$ is the saturation vapor pressure corresponding to a flat liquidvapor interface, $M_{v}$ is the vapor molecular weight, $R$ is the universal gas constant, $T$ is the temperature (assumed equal to $293 \mathrm{~K}$ throughout the paper), $\gamma$ is the surface tension, $\theta$ is the contact angle, $\rho_{\ell}$ is the liquid density, and $r$ is the radius of curvature of the liquidgas interface considered as a spherical cap at the entrance of a cylindrical pore. The relationship expressed in Eq. (1) is classically used for modeling vapor capillary condensation and analyzing the sorption isotherms. ${ }^{5}$

As illustrated in Fig. 1 representing $R H_{\text {equ. }}$ vs $d=2 r$ for water and $\theta=0$, the Kelvin effect is only important for pore sizes smaller than $100 \mathrm{~nm}$, and more specifically for diameters smaller than about $20 \mathrm{~nm}$. This justifies the consideration of the Kelvin effect for modeling the drying process in mesoporous materials. For pore diameters larger than $100 \mathrm{~nm}$, the effect becomes gradually negligible and the equilibrium vapor pressure is nearly equal to the saturation vapor pressure. However, the lower bound of applicability of the Kelvin relationship in terms of the pore diameter remains uncertain. A minimum diameter of $4 \mathrm{~nm}$ is often considered (see, for instance, Ref. 22), and accuracy of Eq. (1) may be questionable below this value. In the remainder of this work, pore diameters not smaller than $4 \mathrm{~nm}$ will, hence, be considered in the simulations.

The development of drying PNM including Kelvin effect pertinent for material featuring pores in the mesoscopic range of diameters is proposed in this article, which is organized as follows. The main assumptions are presented in Sec. II, while the pore network structure is briefly discussed in Sec. III. The drying PNM algorithm with Kelvin effect is presented in Sec. IV. Simulations are reported and discussed in Sec. V. Section VI is dedicated to a summary and conclusion of the analysis, and some possible future works are presented.

\section{MAIN ASSUMPTIONS}

Throughout this work, the following assumptions are made regarding the drying process under consideration:

- the network is non-deformable;

- thermal effects are neglected on the ground that only slow drying is considered; 


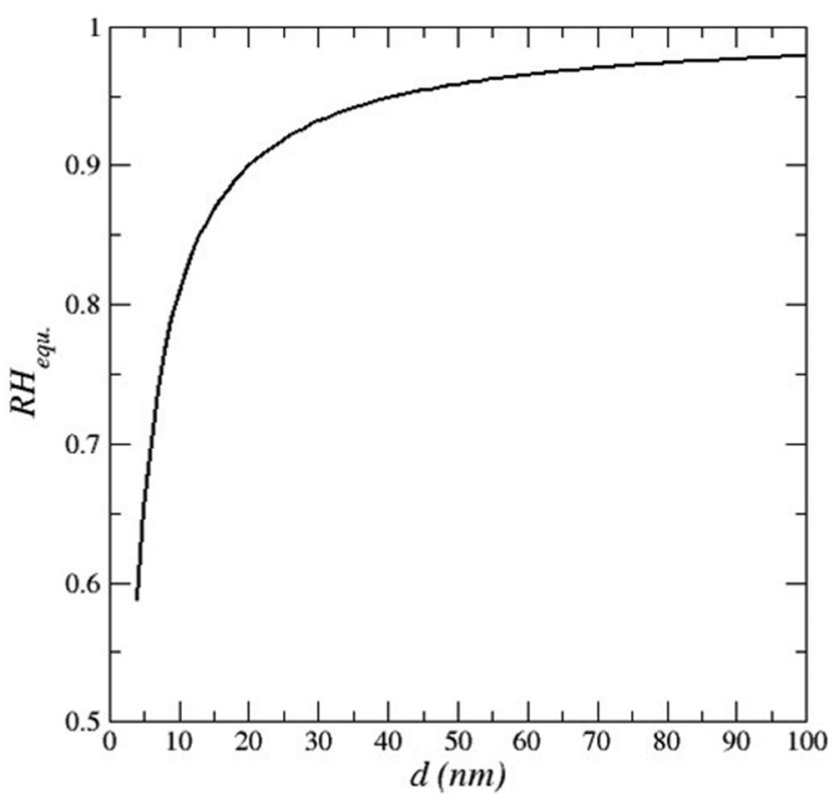

FIG. 1. Variation of water activity (equilibrium relative humidity $R H_{\text {equ. }}$ ) as a function of pore equivalent diameter $d$ according to Kelvin relationship assuming $\theta=0$ and a meniscus at maximum curvature at the pore entrance of diameter $d=2 r$.

- the pressure in the gas phase remains uniform and constant, equal to the atmospheric pressure. This is also because only slow drying processes are considered.

- gravity effects are neglected. As a consequence of the range of pore size under concern, body forces can be expected to be negligible compared to viscous and/or capillary effects.

- the liquid is a perfectly wetting fluid $(\theta=0)$;

According to the literature review presented in the introduction, an important issue lies in the consideration of the liquid films. Two main types of liquid films can be distinguished: the adsorbed films, also referred to as thin films or bound water, and the corner films. The later are driven by capillary effects associated with the presence of corners in the pore space (a typical example is when the pores are of polygonal cross section). The corner films can quite significantly enhance the evaporation compared to a pure vapor diffusion scenario, as shown both for mesoporous ${ }^{16}$ or macroporous systems. ${ }^{15,23,24}$ In this work, corner films are not considered. In other words, it is assumed that the pore space geometry is such that corner films cannot develop or not sufficiently for having a significant impact. The impact of the transport in thin films on evaporation seems less clear as discussed earlier. Clarifying this issue certainly requires specific studies. In the mean time, the following view is adopted. Thin films are considered to be present everywhere in the pore space, but the transport in the thin films is neglected. Since the literature review suggests that the transport in thin film is not necessarily negligible compared to vapor diffusion, the latter assumption will have to be reconsidered in a future work. As also implicitly indicated in Ref. 6, it is considered that the bound water thickness simply adjusts to the local vapor pressure. As reported in Ref. 8, bound water film typical thickness is on the order of $1 \mathrm{~nm}$. Thus, even if the bound water transport is negligible, the presence of the bound water reduces the effective size of the pores as regards the space left free for vapor diffusion and the meniscus maximum curvature computation. Based on these elements, it is assumed that all the pore sizes considered in what follows are effective pore sizes, i.e., the real pore size minus twice the bound water film thickness. As suggested in Ref. 6, it is actually expected that the combination of the thin film transport, if any, and the vapor diffusion transport in the pores could be formulated as an effective diffusion problem with the effective diffusion coefficient greater than for the pure vapor diffusion. In this case, the model presented in what follows could be easily adapted and the main modeling point addressed in what follows, i.e., the impact of the liquid phase fragmentation and the menisci curvature radius computation at the boundary of each cluster, would be still relevant.

An additional issue lies in the condition to be applied at the menisci. The interfacial mass flux at a meniscus is described by the Schrage equation derived from kinetic theory, ${ }^{25}$ which takes into account the fact that the conditions at an evaporating meniscus deviate from thermodynamic equilibrium conditions. However, as discussed for instance in Ref. 26, the additional interfacial resistance associated with this non-equilibrium effect is actually negligible compared to the resistance due to transport phenomena in most practical situations. In other words, the evaporation is controlled by the transport phenomena in the situation considered in the present article and does not depend on the interfacial resistance. For this reason, the classical local thermodynamic equilibrium assumption is used to specify the conditions at the menisci.

To summarize, the phenomena taken into account in the present model are the vapor diffusion, including Knudsen effect, in the gas phase, the capillary effects, and the Kelvin effect.

\section{PORE NETWORK MODEL}

While using PNM, two types of pore networks can be usually distinguished, namely structured and unstructured networks. ${ }^{27}$ For simplicity, and as in most previous works on drying PNM, a structured model is considered here. It is made of a square network in $2 \mathrm{D}$, as depicted in Fig. 2.

In this type of model, the pore space is conceptualized as a network of pore bodies located at the nodes (sites) of the network connected by smaller channels, or throats, corresponding to the constrictions between pore bodies. These channels correspond to the network bonds. Different options have been considered to distribute the pore space between the pore bodies (sites) and the channels (bonds). For instance, volumeless pore bodies were considered in Ref. 28 , the pore space volume being only within the bonds. Alternatively, the pore space volume was distributed between pore bodies and bonds in Ref. 19, whereas volumeless throats (bonds) were considered in Ref. 29 , the pore space volume being only present in the pore bodies. In what follows, volumes are assigned to both throats and pore bodies as in Ref. 19.

Spherical pore bodies of diameters $d_{p}$ are placed at the network nodes. The lattice spacing, i.e., the distance between two neighboring pore bodies, is denoted by $a$. In the present work, this parameter is kept constant and is set to $a=300 \mathrm{~nm}$. The pore body diameter is randomly distributed according to a given probability density function (p.d.f.) in the range $\left[d_{\text {pmin }}, d_{\text {pmax }}\right]$. Bonds are cylindrical channels 


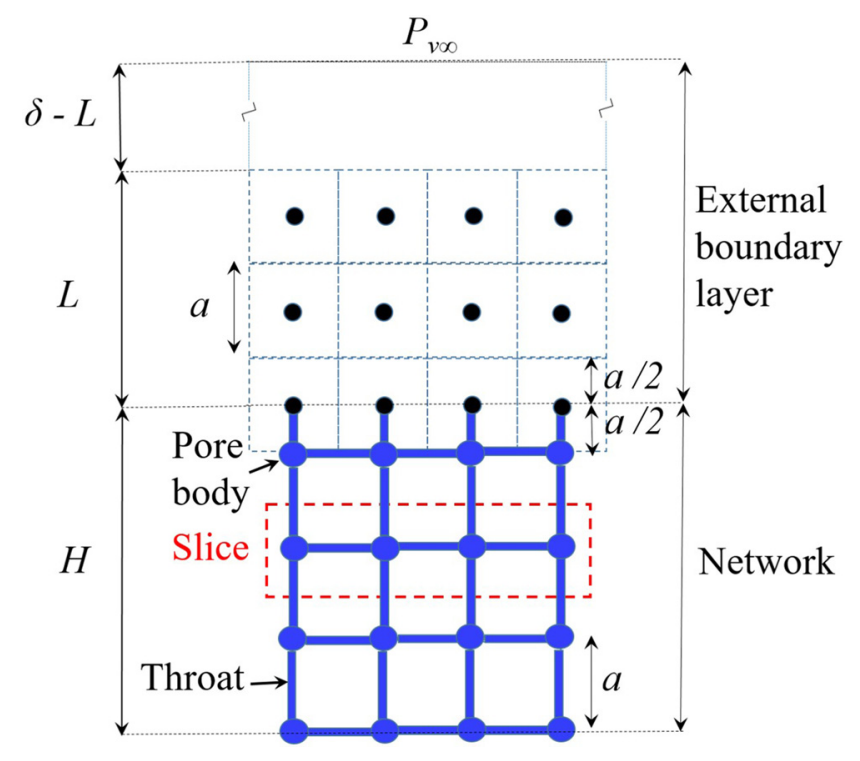

FIG. 2. Square pore network. The red dashed box corresponds to an exemplary network slice used for spatially averaging the PNM simulation data. Black dots correspond to computational nodes in the sub-layer of the external boundary layer.

characterized by their diameter $d_{t}$, which is also randomly distributed according to a given p.d.f. in the range $\left[d_{t \text { min }}, d_{t \max }\right]$ with the constraint that the size of a bond is smaller than the smallest size of adjacent pore bodies. In the present analysis, uniform p.d.f. is considered. The pore body (the throat, respectively) size distribution is referred to as the PSD (the TSD, respectively).

\section{DRYING ALGORITHM}

The drying PNM presented in what follows can be seen as an extension of the model proposed earlier. ${ }^{19}$ A key feature of the model is the full consideration of the fact that many liquid clusters can form during the drying process. Also, the model is based on the application of the invasion percolation (IP) throat selection rule to each cluster. ${ }^{30}$ It has been shown that the IP rules are still valid in mesoporous materials. ${ }^{31,32}$ This model is summarized later. The new features introduced for the present study are described afterward.

At a given time during drying, a pore body or a throat can be either partially or fully invaded by the gas phase. As sketched in Fig. 3, one can distinguish five situations regarding the pore bodies (or throats):

- pore bodies of type L, fully filled by liquid. All the four adjacent bonds are also saturated by the liquid phase;
- pore bodies of type PI, i.e., partially invaded by the gas phase. Pore bodies PI contain a liquid/gas interface and are connected to only one gas saturated adjacent bond;

- pore bodies of type CI, i.e., completely invaded, correspond to pore bodies containing gas only but connected to at least one liquid adjacent bond;

- pore bodies of type IF, i.e., interfacial pore bodies, correspond to pore bodies containing liquid only but connected to only one gas saturated adjacent bond. In a cluster, only one IF pore body may exist. However, IF bonds may be multiple in a cluster;

- pore bodies of type G, containing only gas and with all connected gas filled adjacent bonds.

The drying algorithm can be summarized as follows.

(1) Each liquid cluster in the network is identified, and a label is attributed to them. This label is also given to each pore body and throat belonging to the cluster under consideration. This task is performed using the deep-search algorithm (see, e.g., Ref. 33)

(2) For clusters that include a PI pore body or throat, this pore body or throat is directly selected as the one to be invaded for this cluster. For the other clusters, the IF pore body or the IF bond of smallest capillary pressure threshold is identified. The capillary pressure threshold of a bond of diameter $d_{t}$ is expressed according to Laplace's law as

$$
p_{c a p} \approx \frac{4 \gamma \cos \theta}{d_{t}}
$$

Thus, $p_{c a p}$ is the minimum pressure difference between the two fluids allowing the bond to be invaded by the gas phase. From Eq. (2), it is obvious that the selected IF bond in a given cluster is one of largest diameter located at the cluster boundary. As pore body sizes are greater than bond sizes, if there exists an IF pore body, it is directly selected. Otherwise, the IF bond with the biggest diameter is identified.

(3) The diameter of the selected pore body or bond in cluster \# $n$ is denoted by $d_{s n}$. The volume of liquid in this throat or pore body at the considered time is $V_{s n}$. If the selected pore body (or throat) is a PI pore body (or throat), then $V_{s n}$ is the volume of liquid remaining in the PI pore body (or throat). This choice is a consequence of the quasi-static approach. Since viscous effects are neglected, the pressure field is spatially uniform in the gas phase and in each liquid cluster. This implies that all the menisci located at a cluster boundary have the same curvature.

(4) The vapor partial pressure field is computed in the gas phase solving numerically the linear system resulting from the modeling of the diffusive transport (see later).

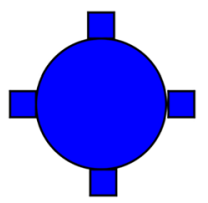

pore body $\mathrm{L}$

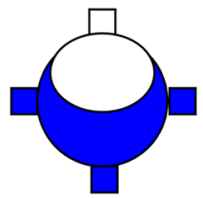

pore body PI

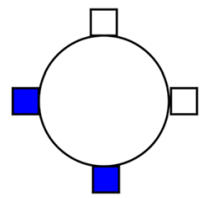

pore body CI

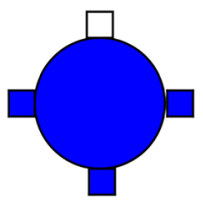

pore body IF

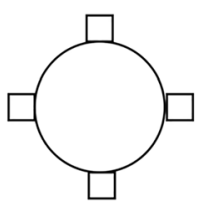

pore body $\mathrm{G}$

FIG. 3. Sketch of various pore body types considered in the PNM (liquid in blue and gas in white). 
(5) The evaporation rate, $F_{n}$, at the boundary of each cluster is computed from the vapor partial pressure field computed in step (4) as

$$
F_{n}=\sum_{k} \sum_{j} \phi_{k j}
$$

where $\phi_{k j}$ is the evaporation rate through the interfacial bond connecting any pore body $k$ at the boundary within the cluster (this can be a L, PI, or IF pore body) to a gas saturated pore body $j$ outside the cluster (i.e., a CI or G pore body). The expression of $\phi_{k j}$ is detailed later [see Eqs. (4) and (8)].

(6) The time, $t_{n}^{*}$, needed to fully evaporate the liquid volume, $V_{s n}$, is computed for each cluster as $t_{n}^{*}=\rho_{\ell} V_{s n} / F_{n}$.

(7) The pore body or throat identified in step (2) for which the corresponding invading time is minimum, $t_{c \min }^{*}=\min \left(t_{n}^{*}\right)$, is fully invaded.

(8) The total elapsed time is computed as $t=t+t_{c m i n}^{*}$.

(9) The phase distribution in the network is updated, including the partial drainage/evaporation of the liquid contained in the other elements (throats or PI pore bodies) identified in step 2, so that $V_{s n}\left(t+t_{c \text { min }}\right)=V_{s n}(t)-\frac{F_{n}}{\rho_{\ell}} t_{c m i n}$.

Compared to the algorithm for capillary porous media, ${ }^{19}$ two modifications are introduced. The first one is straightforward and is related to the modeling of the vapor diffusion transport in small pores while considering the Knudsen diffusion. The second one is more important and is directly related to the consideration of the Kelvin effect. To begin with, modeling of the vapor diffusion transport shall be detailed.

The classical assumption to model diffusive transport of vapor in the gas phase is to make use of the quasi-steady assumption. This is based on the observation that the characteristic time of drying, i.e., the time needed to reach the final liquid distribution in the network, is large compared to the characteristic time of diffusion at the pore scale. The mass rate between pore body $i$ and pore body $j$ fully occupied by the gas phase (pore bodies $\mathrm{G}$ or $\mathrm{CI}$ ) through the bond connecting them is expressed using Fick's law as

$$
\phi_{i j}=D_{e f f} \frac{M_{v}}{R T} \frac{\pi d_{t i j}^{2}}{4}\left(\frac{P_{v j}-P_{v i}}{a}\right) .
$$

Here, $P_{v}$ is the vapor partial pressure and $D_{\text {eff }}$ is computed according to the following relationship: ${ }^{34}$

$$
\frac{1}{D_{e f f}}=\frac{1}{D_{v}}+\frac{1}{D_{K n u}},
$$

where $D_{v}$ is the vapor molecular diffusion coefficient and $D_{K n u}$ is the Knudsen diffusion coefficient. The Knudsen diffusion is considered here because the pore diameters are not large compared to the gas molecule mean free path. ${ }^{35}$ The Knudsen diffusion coefficient is expressed as

$$
D_{K n u}=\frac{d_{t i j}}{3} \sqrt{\frac{8 R T}{\pi M_{v}}} .
$$

A linear system of equations is obtained by expressing the mass conservation in each pore body $i$ filled with gas (pore bodies $G$ or $\mathrm{CI}$ ),

$$
\sum_{j} \phi_{i j}=0
$$

where index $j$ refers to the neighbor pore bodies connected to pore body $i$ by a gaseous bond. The resulting linear system is solved numerically to obtain the partial pressure at each gaseous or partially gaseous node of the network. However, to do so, boundary conditions must be specified, and this is performed as follows. Let us assume that the radius of curvature of each meniscus is known. The method for its determination is a crucial new feature of the present PNM, and the way to compute it is described right after.

When pore body $i$ is of PI type, the vapor partial pressure is specified in this pore body from the Kelvin relationship [Eq. (1)] using the radius of curvature of the meniscus present in this pore body. Thus, the vapor partial pressure in such pore body is not obtained from the solution of the linear system but is specified as a (Dirichlet) boundary condition.

When pore body $i$ is of CI type, the evaporation rate at a meniscus located at the entrance of an adjacent liquid throat (connecting pore body $i$ to pore body $j$ ) is expressed as

$$
\left.\phi_{i j}=D_{\text {eff }} \frac{M_{v}}{R T} \frac{\pi d_{t i j}^{2}}{4} \frac{P_{v e q u . j}-P_{v i}}{\left(d_{p i} / 2\right)}\right) .
$$

The vapor partial pressure $P_{\text {vequ.j }}$ at the meniscus under consideration is determined from the Kelvin relationship using the radius of curvature of that meniscus. In Eq. (8), $d_{p i} / 2$ corresponds to the distance between the meniscus and the pore body center, whereas $D_{\text {eff }}$ is computed by replacing $d_{t i j}$ by $d_{p i}$ in Eq. (6).

The last step to form the linear system allowing the computation of the vapor partial pressure in each gaseous node of the network is to specify the boundary conditions at the surfaces limiting the porous domain. A zero flux condition is imposed on the impervious surfaces, i.e., at the network lateral and bottom surfaces for the simulations carried out in this work. Then, the boundary conditions at the open surface, i.e., the surface through which the vapor can exit the porous medium, also need to be specified. In the present case, only the network top surface is open. To couple the network with the external gas, vapor partial pressure computational nodes are added on top of the network as sketched in Fig. 2. As indicated in this figure, the vapor partial pressure is imposed $\left(P_{v}=P_{v \infty}\right)$ at a distance $\delta$ from the network surface. This condition on the relative humidity is denoted as $R H_{\infty}=\frac{P_{v \infty}}{P_{v s}}$ in the following. In the layer of size $L$ within $\delta$, the mass flux between two nodes is given by an expression similar to Eq. (4), namely,

$$
\phi_{i j}=D_{v} \frac{M_{v}}{R T} a^{2}\left(\frac{P_{v j}-P_{v i}}{a}\right) .
$$

The vertical flux between a node in the upper row of nodes within the layer $L$ and the upper boundary where $P_{v}=P_{v \infty}$ is expressed as

$$
\phi_{i j}=D_{v} \frac{M_{v}}{R T} a^{2}\left(\frac{P_{v \infty}-P_{v i}}{\frac{a}{2}+\delta-L}\right) .
$$

For the nodes located at the interface between the network and the external diffusive layer (Fig. 2), the vapor partial pressure is specified 
from the Kelvin relationship when the adjacent vertical throat is liquid. When the throat is gas saturated, the expression of the flux is given by Eq. (4).

The use of an external diffusive layer made of a layer of thickness $L$ which is discretized using the same mesh size, $a$, and the layer of thickness $\delta$ - $L$ is motivated by the fact that it is of interest to account for situations where $\delta \gg a$, representative of cases encountered in the porosimetry technique called evapo-porosimetry. ${ }^{36}$ Actually, at the pore network surface and in its close neighborhood, the vapor partial pressure field and the diffusive flux remain heterogeneous. The heterogeneity is then gradually damped out with an increasing distance from the surface. This explains why it is not necessary to set computational nodes all over the diffusive layer of thickness $\delta$. In the layer sufficiently away from the surface, the diffusive transfer is unidirectional and an analytical formula, as given by Eq. (10), can then be used to model the transfer.

\section{A. Kelvin effect implementation}

As described earlier, the drying algorithm requires the determination of the menisci curvature at the boundary of each liquid cluster at each step of the drying process since this information is needed for computing the equilibrium vapor pressure from the Kelvin relationship. Computation of the curvature is actually a tricky part of the algorithm.

As simplified approaches, it could be appealing to first consider two options. Since the largest throat at the boundary of a cluster is the one to be invaded in the capillary limit envisaged in this work, the first obvious option, referred to as the "simple option," is to consider that the vapor pressure at the boundary of a cluster is given by the Kelvin relationship with $r=d_{\text {tmax }} / 2$, where $d_{\text {tmax }}$ is the diameter of the largest interfacial throat. However, since $d_{\text {tmax }}$ varies from one cluster to the other, the vapor partial pressure at the boundary also differs from one cluster to the other. The net result of this procedure is the generation of a negative mass transfer rate, $F_{n}$, i.e., "condensation," at the boundary of some clusters. This situation is illustrated in Fig. 4(a). As a consequence, clusters featuring a negative mass transfer rate would grow, and, therefore, the menisci radius of curvature would be modified at their boundaries. This is in contradiction with the initial assumption that $r=d_{\text {tmax }} / 2$. In other words, the assumption that the radius of curvature is simply given by $d_{\text {tmax }} / 2$ does not lead to consistent results. A simple tempting correction, as a second option, is then to set $F_{n}$ to zero for such clusters, for instance imposing a zero-flux condition at each meniscus of the cluster. As illustrated in Fig. 4(b), this also leads to inconsistency as negative mass rate may result at new clusters. Typically, with this option, the vapor partial pressure varies along the boundary of the cluster, which is not consistent with the Kelvin relationship combined with the capillary equilibrium condition, imposing that the meniscus curvature is the same for all the menisci belonging to the boundary of a given cluster. A more refined and physically consistent approach is hence needed. This means that the menisci curvature radius at the boundary of each cluster must be determined, so that the evaporation rate $F_{n}$ remains $\geq 0$ for each cluster. The procedure implemented in the algorithm for this purpose is the following.

Basically, the idea for eliminating the negative fluxes is to determine the equilibrium vapor pressure at the boundary of each cluster so that a zero or positive net overall flux is obtained, i.e., such that $F_{n}$ $\geq 0$, for each cluster, including those formed by a single liquid throat. Contrary to the simpler (but inconsistent) procedure consisting in imposing a zero flux at each meniscus of the cluster, a zero overall flux is now achieved by exactly compensating condensation at some menisci by evaporation from the other menisci belonging to the same cluster. More specifically, the vapor pressure at the cluster boundary is obtained by using the following procedure. Let us assume that $n$ clusters with negative fluxes are identified among the $N$ clusters present in the network. The vector, $\mathbf{F}_{\mathbf{n}}$, of negative mass transfer rate components $F_{n i}\left(P_{n i}\right)$, where $P_{n i}$ is the equilibrium vapor pressure for cluster $\# i(1 \leq i \leq n)$, must be changed to zero. Newton's method is used to obtain the zero of vector $\mathbf{F}_{\mathbf{n}}$. It is an iterative method that consists in imposing the zero flux condition through the following relationship:

$$
\mathbf{P}_{\mathbf{n} k}=\mathbf{P}_{\mathbf{n} k-1}-\mathbf{J}_{\mathbf{n} k}^{-1} \mathbf{F}_{\mathbf{n} k},
$$

where the subscript $k$ denotes the $k$ th iteration, $\mathbf{P}_{\mathbf{n}}$ is the equilibrium vapor pressure vector (of components $P_{n i}, 1 \leq i \leq n$ ) for the $n$ clusters, $\mathbf{J}_{\mathbf{n}}$ is the Jacobian matrix of size $n \times n$, and components $J_{n i j}$ defined as

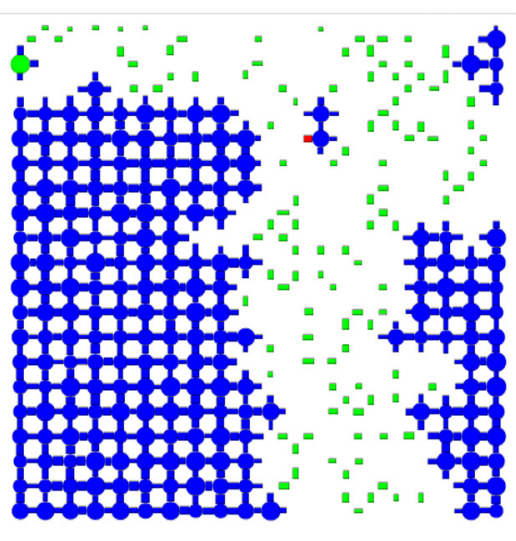

(a)

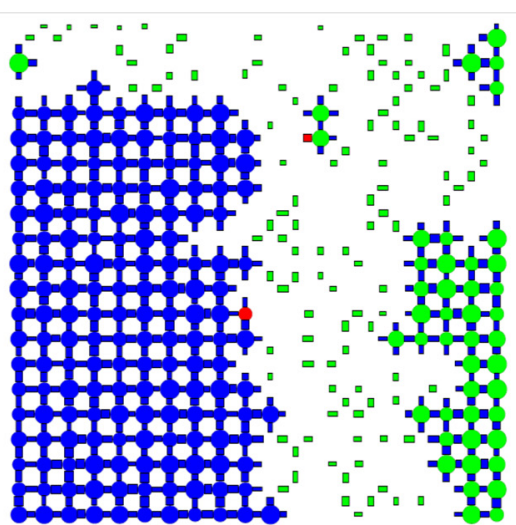

(b)
FIG. 4. (a) Example of a situation where a negative mass transfer rate is obtained for a cluster (in green) when the simple option (see the text) is used. (b) If a zero evaporation rate is imposed at each interfacial throat of a cluster with a negative flux, then clusters that were with a positive evaporation rate can become clusters with a negative evaporation rate. Pore bodies and throats in red are $\mathrm{PI}$ (partially invaded) elements; clusters or single throats for which a negative mass transfer rate is computed are shown in green. Vapor escapes from the network top edge. 


$$
J_{n i j}=\frac{\partial F_{n i}}{\partial P_{n j}} .
$$

This matrix is computed using a finite difference method at the $k$ th iteration as

$$
J_{n i j k}=\frac{F_{n i k}-F_{n i k-1}}{P_{n j k}-P_{n j k-1}},
$$

where $F_{n i k}$ and $F_{n i k-1}$ (respectively, $P_{n j k}$ and $P_{n j k-1}$ ) are the mass transfer rate (the equilibrium vapor pressure, respectively) at the boundary of cluster $i$ (cluster $j$, respectively) at iterations $k$ and $k-1$.

In order to obtain the Jacobian matrix at each iteration, the vapor partial pressure field over the whole network must be computed $n$ times. This means that the computational time increases with the number of clusters with negative fluxes. If the iteration number to reach the solution is $m$, then the necessary time is approximatively $m \times n \times t$, where $t$ is the computational time of the vapor partial pressure field. For cluster $i$, the flux $F_{n i}$ is related to the vapor partial pressure field around the cluster according to Eqs. (3) and (8). Because this flux to equilibrium vapor pressure relationship is linear, Newton's method converges with only one iteration, and hence, the computational time is simply $n \times t$.

\section{RESULTS}

Unless otherwise mentioned, results are presented for the following conditions. The network is a $20 \times 20$ square pore network with uniform probability density functions (p.d.f.) for the PSD and the TSD and a lattice spacing $a=300 \mathrm{~nm}$. The pore body radii are in the range $(65-135 \mathrm{~nm})$, whereas the throat radii vary from 2 to $48 \mathrm{~nm}$. The total thickness of the external boundary layer is $\delta=10 \mathrm{~cm}$, which is consistent with the typical configuration encountered in the evapoporosimetry technique. ${ }^{36}$ The external boundary sub-layer, adjacent to the network where computational nodes are set, is of thickness $L=50 a(15 \mu \mathrm{m})$.

In Fig. 5, the drying patterns obtained when the Kelvin effect is taken into account and when it is neglected, i.e., when $P_{v}=P_{v s}$ is imposed at the surface of the menisci, are compared. The external relative humidity is $R H_{\infty}=0$ in these simulations. As can be seen, the Kelvin effect does have an important impact on the pattern.

Whereas a drying zone develops in the top region of the network when Kelvin effect is neglected, it can be seen that this is not the case when the Kelvin effect is taken into account. Because of the Kelvin effect, the evaporation rate of a cluster in the network top region can be quite small. As a result, such a cluster can remain much longer in the top region than in a network where the Kelvin effect is neglected.

It should also be noted that in the presence of the Kelvin effect, many single liquid throats distributed all over the network remain while the drying process progresses. In a capillary porous material, a full drying is expected for any value of the external relative humidity $R H_{\infty}$ lower than 1, except for a tiny volume of liquid confined in thin films, crevices, grain contacts, or wall roughness corresponding to a very small fraction of the pore space. In a hygroscopic material, incomplete drying can be obtained depending on the pore size distribution and $R H_{\infty}$. If the throat diameters are all greater than the "critical" throat diameter, $d_{c}$, given by the Kelvin relationship, i.e., $d_{c}=\frac{M_{v}}{R T} \frac{4 \gamma \cos \theta}{\rho_{\ell} \ln \left(\frac{P_{v e q u}}{R H_{\infty} P_{v s}}\right)}$, then full drying is expected (except for possible
With Kelvin's effect
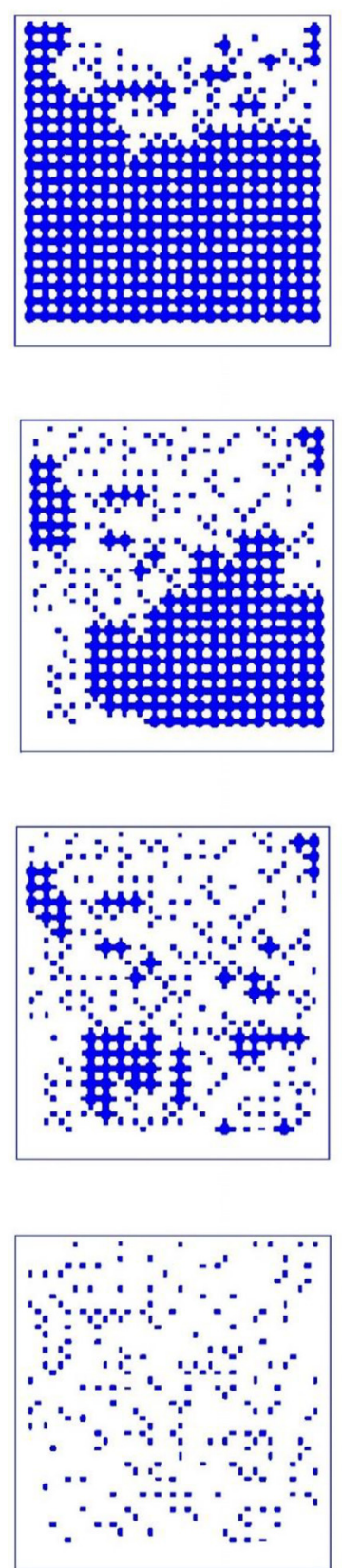

Without Kelvin's effect
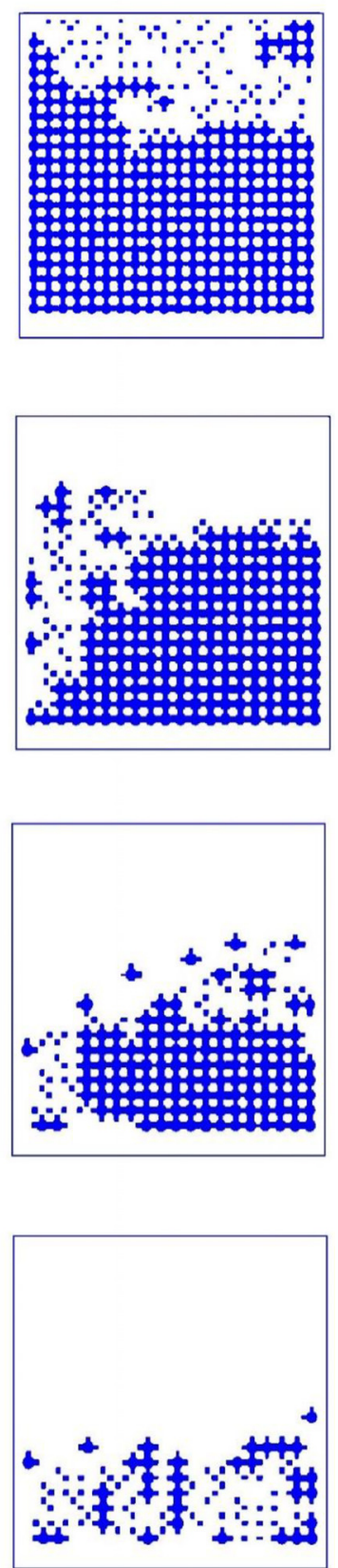

FIG. 5. Liquid (in blue) and gas (in white) distributions in the network for a simulation with Kelvin effect (left) and without Kelvin effect (right). Patterns are shown for the same number of invaded pore bodies and throats. Vapor escapes from the top edge of the network. $R H_{\infty}=0$.

remaining thin liquid films). When a fraction of the throats in the porous material has a diameter smaller than $d_{c}$, drying should be incomplete since this fraction of throats cannot be invaded (in the absence of significant viscous effects as considered in this work). This 
is illustrated in Fig. 6. Naturally, if all the throats are lower than $d_{c}$, then no drying at all occurs.

\section{A. Drying pattern}

\section{With Kelvin's effect without Kelvin's effect}

\section{B. Meniscus curvature distribution}

To characterize the fact that the meniscus curvature varies from one cluster to the other and, thus, also the equilibrium vapor partial pressure (the equilibrium vapor partial pressure is the vapor partial pressure computed at the boundary of a cluster), the average equilibrium vapor partial pressure and its standard deviation distribution were computed as follows:

$$
\bar{P}_{\text {vequ. }}=\frac{1}{N} \sum_{k=1}^{k=N} P_{\text {vequ. }-k},
$$

$$
\sigma_{P_{\text {vequ. }}}=\sqrt{\frac{\sum_{k=1}^{k=N}\left(P_{\text {vequ. }-k}-\bar{P}_{\text {vequ. }}\right)^{2}}{N}},
$$
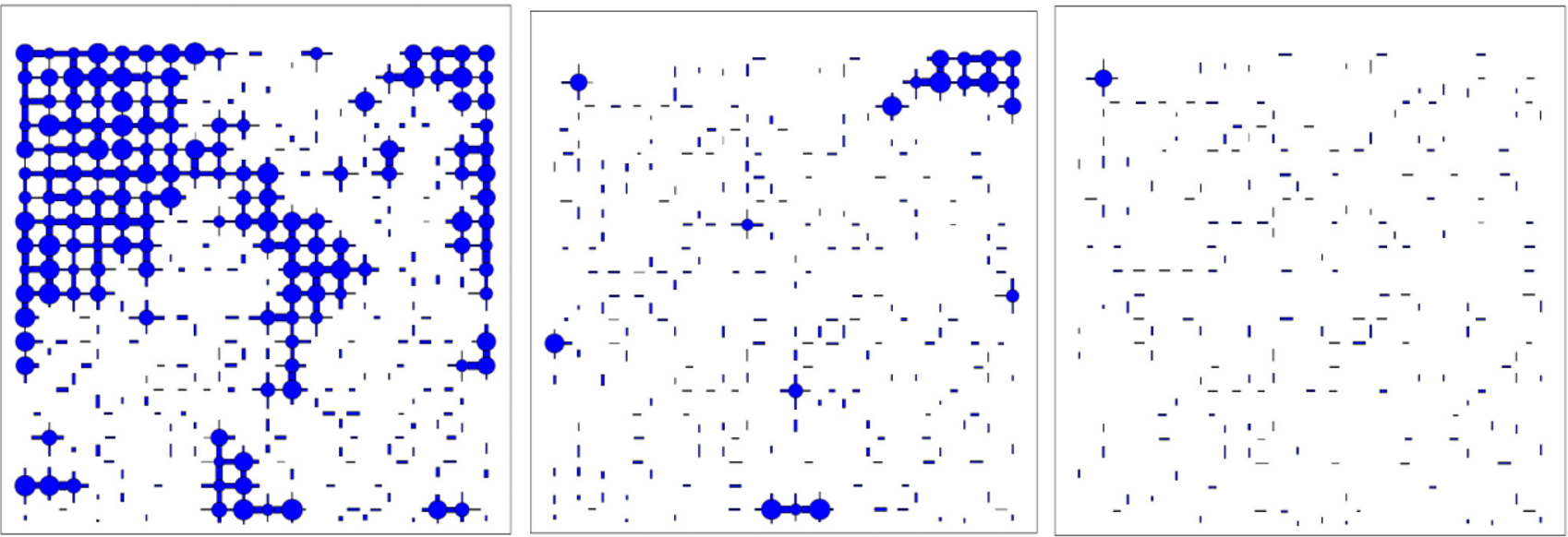

FIG. 6. Final drying patterns for three different values of $R H_{\infty}, 0.96,0.94$, and 0.92 , leading to incomplete final drying. The throat radii are distributed in the range $(2-48 \mathrm{~nm})$.

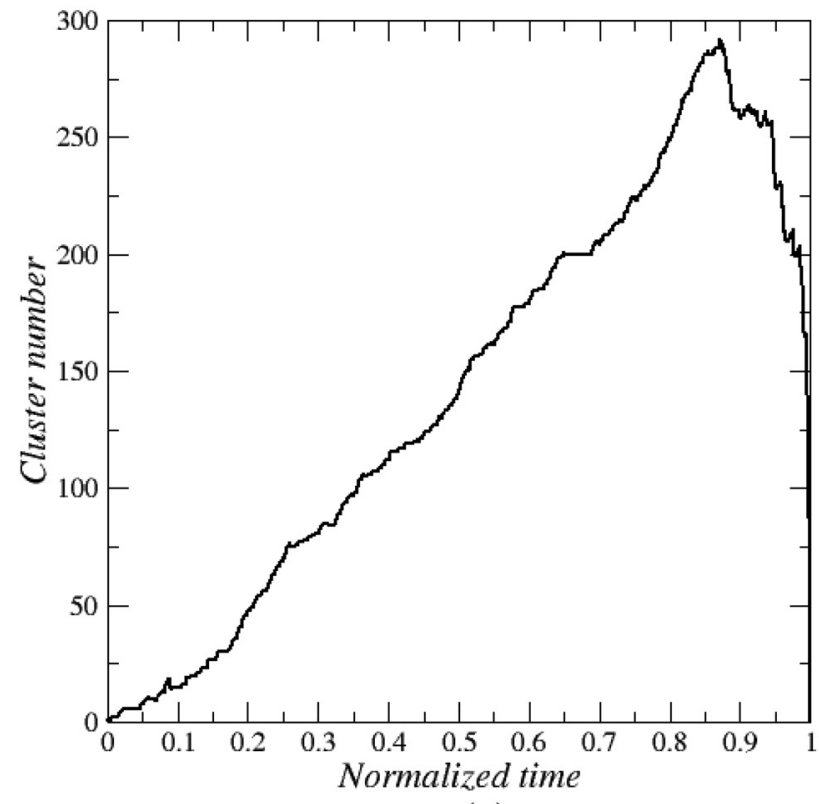

(a)

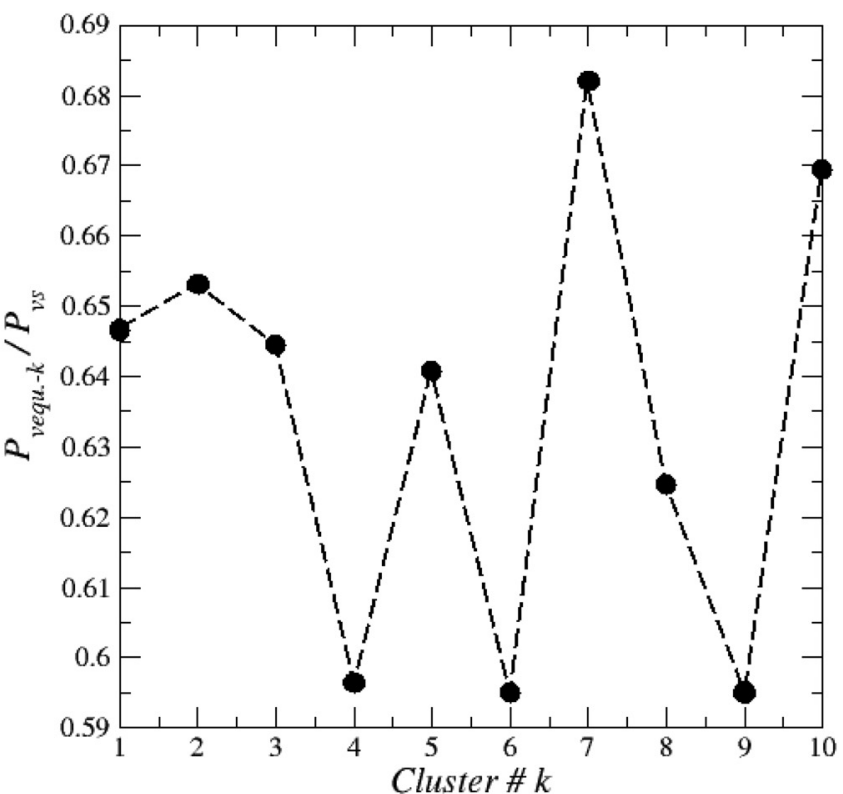

(b)

FIG. 7. (a) Variation of the number of clusters present in the network during the drying process; (b) illustration of the radius of curvature variation from one cluster to the other (case when 10 clusters are still present in the network). 
where $N$ is the number of clusters in the network and $P_{\text {vequ. }-k}$ being the equilibrium vapor partial pressure at the boundary of cluster \#k.

In Fig. 7(a), representing the variation of the number of clusters vs the normalized time, one can distinguish two main periods: a first period during which the number of clusters steadily increases up to a maximum and a second period where the number of clusters present in the network decreases. These two periods are referred to as period 1 and period 2, respectively.

\section{Period 1}
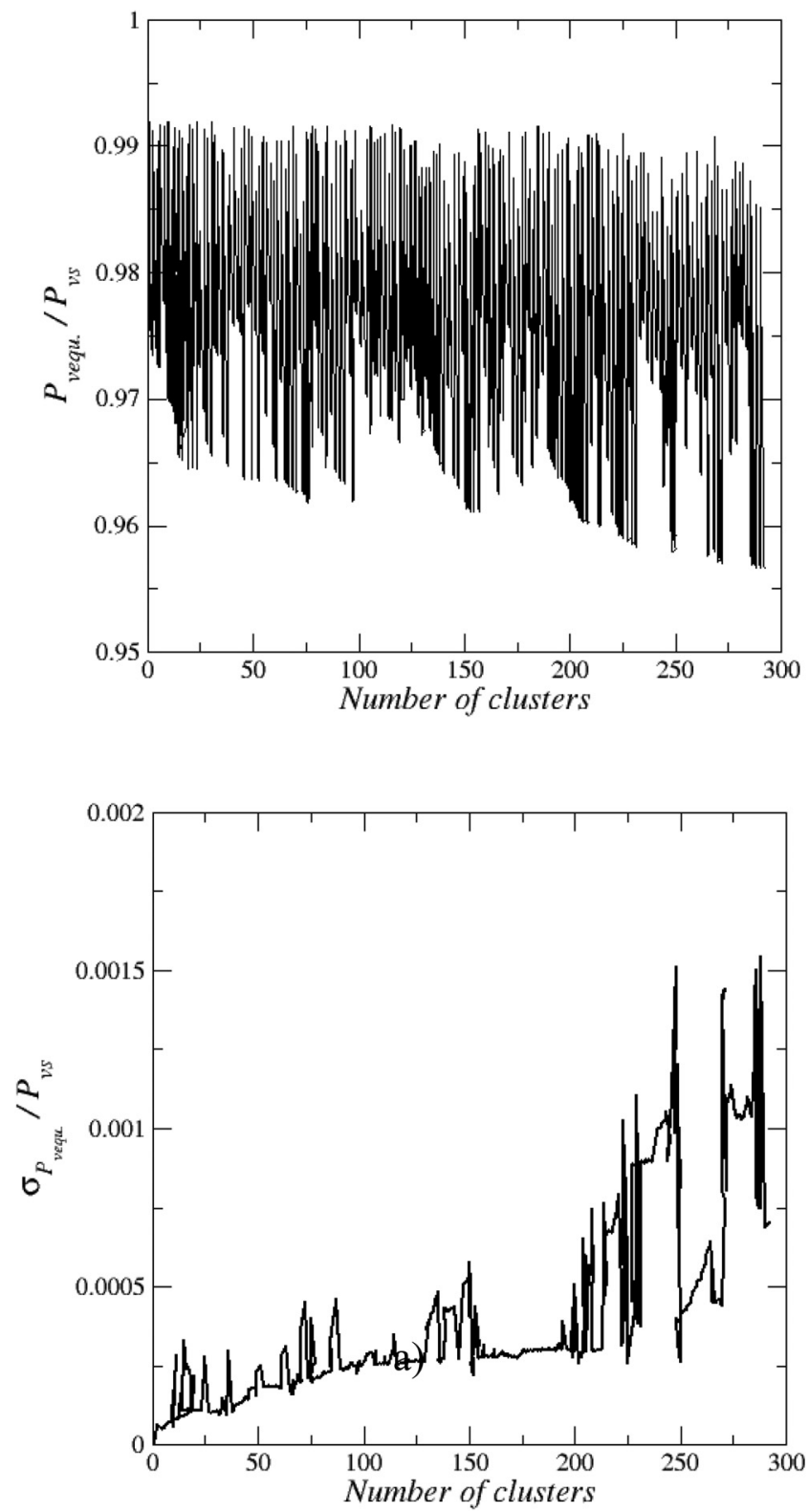

The fact that the equilibrium vapor pressure varies from one cluster to the other is illustrated in Fig. $7(\mathrm{~b})$ for the case where $\sigma_{P_{\text {vequ. }}} / P_{v s}$ is maximum (see Fig. 8). In Fig. 8, variations of the mean equilibrium vapor pressure and standard deviation [Eq. (15)] are represented as functions of the number of clusters present in the network in periods 1 and 2. As can be observed, the mean equilibrium vapor pressure fluctuates with the successive invasions in period 1. In period 2, fluctuations are damped out, and the mean equilibrium vapor pressure gradually decreases with a decreasing

\section{Period 2}
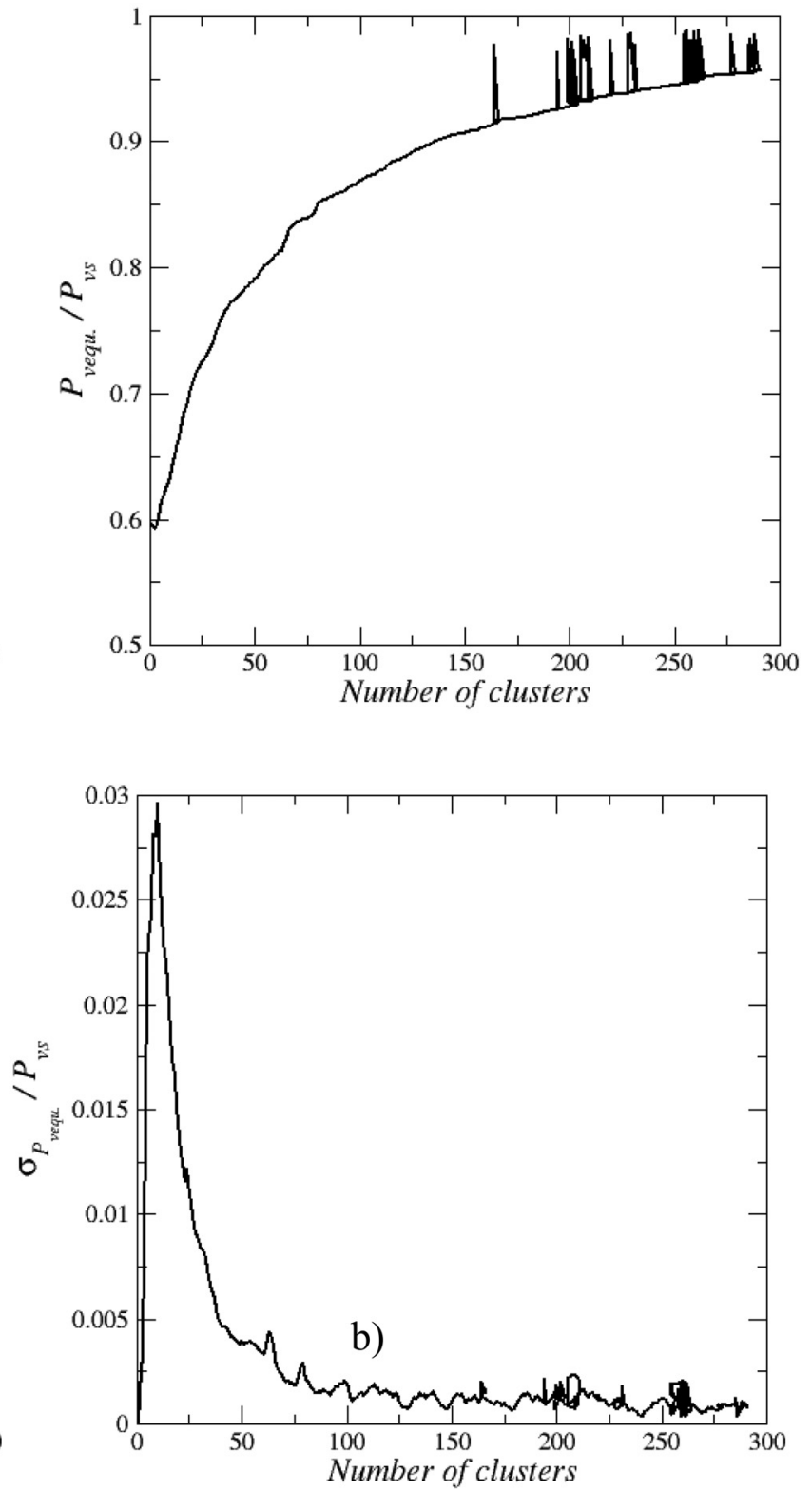

FIG. 8. (a) Variation of the mean equilibrium vapor pressure in the network [Eq. (14)] as a function of the number of clusters in the network in periods 1 and 2; (b) variation of the cluster equilibrium vapor pressure standard deviation [Eq. (15)] as a function of the number of clusters in the network in periods 1 and 2. 
number of clusters present in the network. This can be explained by the fact that only small clusters containing throats of small sizes are present in period 2 (as illustrated in Fig. 5, the liquid is eventually distributed only in narrow throats toward the end of drying). As shown in Fig. 8, the standard deviation [Eq. (15)] increases during most of the drying process until only a few clusters are present (10 in this example).

\section{Drying kinetics}

The drying kinetics in the presence of Kelvin effect and when the Kelvin effect is neglected is compared in Fig. 9 for two values of the external relative humidity, namely, $R H_{\infty}=0$ and $R H_{\infty}=0.6$.

As illustrated in Fig. 9, drying in the presence of Kelvin effect can be faster than drying without Kelvin effect, and the impact is not very sensitive to the applied relative humidity in the external gas phase [see Figs. 9(a) and 9(b)]. It should be noted here that the conditions are exactly the same in both cases, in particular regarding the network which has the same pore body and throat size distributions. Such a comparison is practically impossible to perform experimentally since the Kelvin effect is directly related to the size of the pore bodies and throats. One option could be to vary the contact angle [strong Kelvin effect with a perfect wetting liquid vs a weaker Kelvin effect for a larger contact angle according to Eq. (1)] but then other factors, such as the effect of liquid films ${ }^{15,16}$ or the impact of contact angle on the drying pattern, ${ }^{37,38}$ might affect the comparison. In any case, the comparison shown in Fig. 9 may be considered as paradoxical at first glance since the Kelvin effect reduces the vapor pressure at the menisci surface. Based on this observation, one would expect a slower drying in the presence of the Kelvin effect. However, the faster drying observed in the presence of the Kelvin effect is fully consistent with the patterns

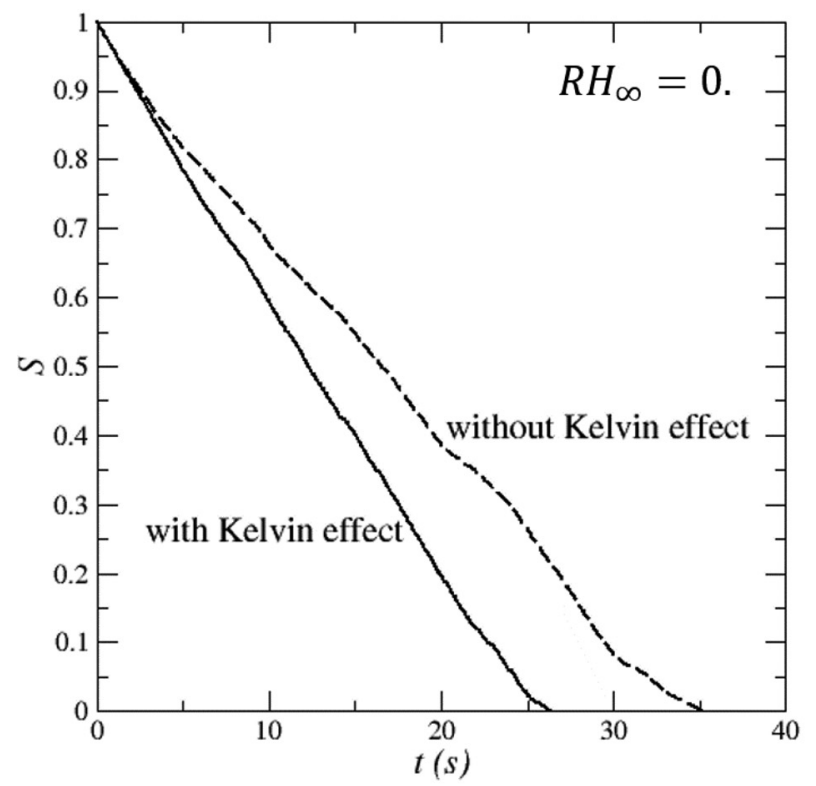

(a) depicted in Fig. 5. Indeed, this figure shows that the liquid phase is more evenly distributed, leading to a larger number of menisci exposed to evaporation in the presence of the Kelvin effect, whereas a dry zone develops in the upper region of the network when the Kelvin effect is not considered. The development of this dry zone is the main phenomenon explaining the slower drying when the Kelvin effect is neglected in the simulation.

In Fig. 10, the total evaporation from the network vs time is reported. This figure highlights the effect of Knudsen diffusion, which reduces the evaporation rate compared to the case where only molecular diffusion is taken into account. This tendency was expected since the effective vapor diffusion coefficient, as expressed in Eq. (5), decreases with an increasing Knudsen diffusion effect. As illustrated in Fig. 10, the impact is quite significant.

\section{Evaporation rate fluctuations}

As depicted in Fig. 10, the evaporation rate fluctuates as a function of time. This is also a distinctive feature compared to drying PNM simulations when the Kelvin effect is neglected. In the latter, the evaporation rate can only decrease. In the presence of a noticeable Kelvin effect, the vapor pressure at the boundary of each cluster fluctuates because of the menisci curvature fluctuations along the drying process (as illustrated in Fig. 8). As a result, when, for instance, a larger throat becomes an interfacial throat, the menisci curvature decreases, and therefore, the menisci vapor pressure increases according to the Kelvin relationship. This, in turn, induces a larger evaporation rate.

\section{E. Desorption isotherm}

The desorption isotherm corresponds to the successive equilibrium saturations obtained when the relative humidity in the external

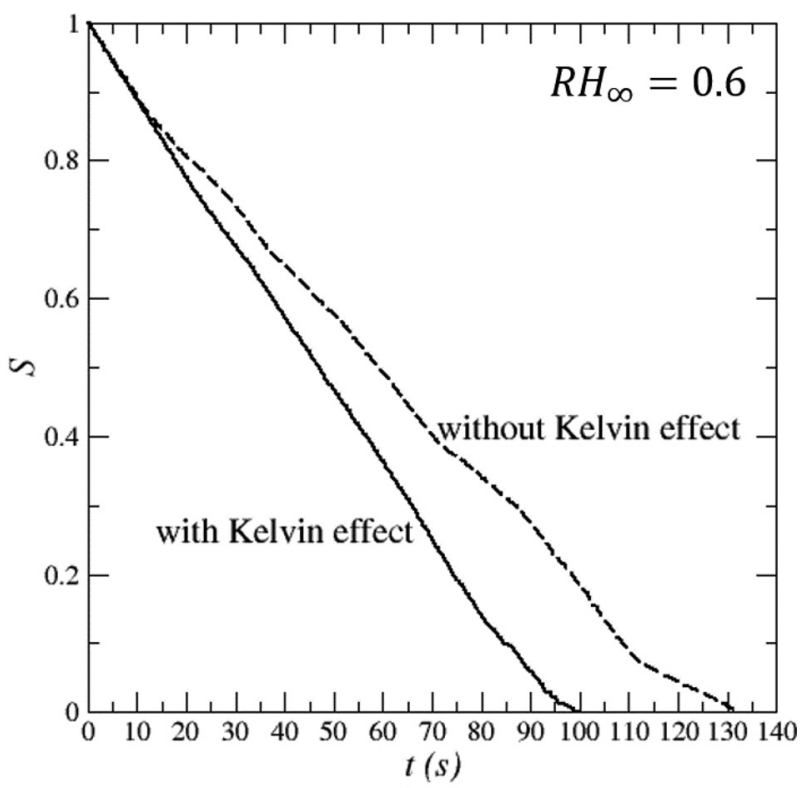

(b)

FIG. 9. Variation of overall network saturation, $S$, as a function of time, $t$, for a simulation with Kelvin effect and without Kelvin effect for (a) $R H_{\infty}=0$ and (b) $R H_{\infty}=0.6$. 


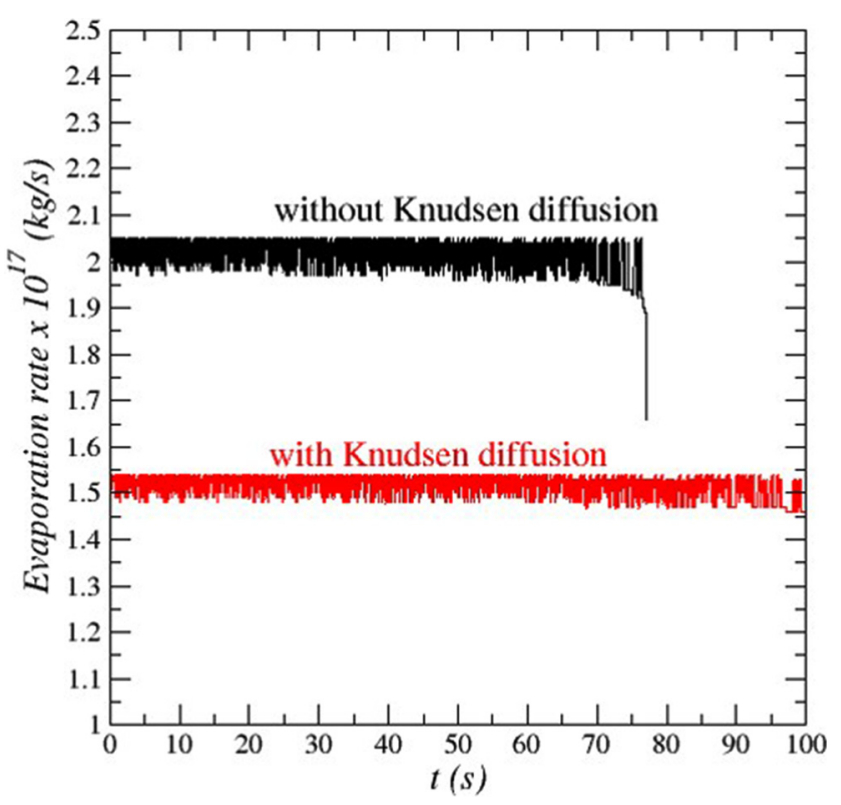

FIG. 10. Impact of Knudsen diffusion on the evaporation rate for a $20 \times 20$ square network. The external relative humidity is fixed to $R H_{\infty}=0$ at $\delta=10 \mathrm{~cm}$. For this simulation, the pore body radii are in the range $(60-100 \mathrm{~nm})$, whereas the throat radii vary from 25 to $55 \mathrm{~nm}$, both following a uniform p.d.f.

gas, $R H_{\infty}$, is gradually decreased with small increments, step by step.

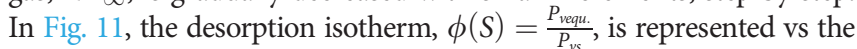
overall network saturation, $S$. This result was obtained for a $20 \times 20$ square network and the nominal conditions, namely, a diffusion layer thickness $\delta=10 \mathrm{~cm}$, pore body and throat radii in the range $(65-135 \mathrm{~nm})$ and $(2-48 \mathrm{~nm})$, respectively, both following uniform p.d.f. The external relative humidity, $R H_{\infty}$, is initially fixed equal to 1 and decreased with a step of 0.02 down to $R H_{\infty}=0.6$. At this value of $R H_{\infty}$, all the liquid in the network has evaporated.

In the limit $\delta \gg H$ ( $H$ denotes the network thickness, see Fig. 2), it is expected that this curve can be obtained in a simpler manner according to the following algorithm, ${ }^{39}$ which does not require solving the diffusion problem

(1) The network is initially fully saturated and the external relative humidity is set to 1 , i.e., $R H_{\infty-0}=1$.

(2) The external gas relative humidity is decreased $R H_{\infty-k+1}=R H_{\infty-k}$ $-\Delta R H$, where $\Delta R H$ is a small relative humidity variation.

(3) Kelvin equation, Eq. (1), is used to determine the critical diameter $d\left(R H_{\infty-k+1}\right)$. All liquid pore bodies and throats having a diameter larger than $d\left(R H_{\infty-k+1}\right)$ can be potentially invaded.

(4) All the liquid pore bodies and throats identified in step 3 and accessible, i.e., connected to the open surface of the network by a gaseous path, are identified.

(5) The pore bodies and throats identified in step 4 are invaded.

(6) Steps 4 and 5 are repeated until no liquid pore body or throat which the diameter is greater than the critical diameter determined in step 3 is present and is accessible.

(7) The network saturation is determined.

(8) Steps 2-7 are repeated until $R H_{\infty-k+1} \leq 0$.

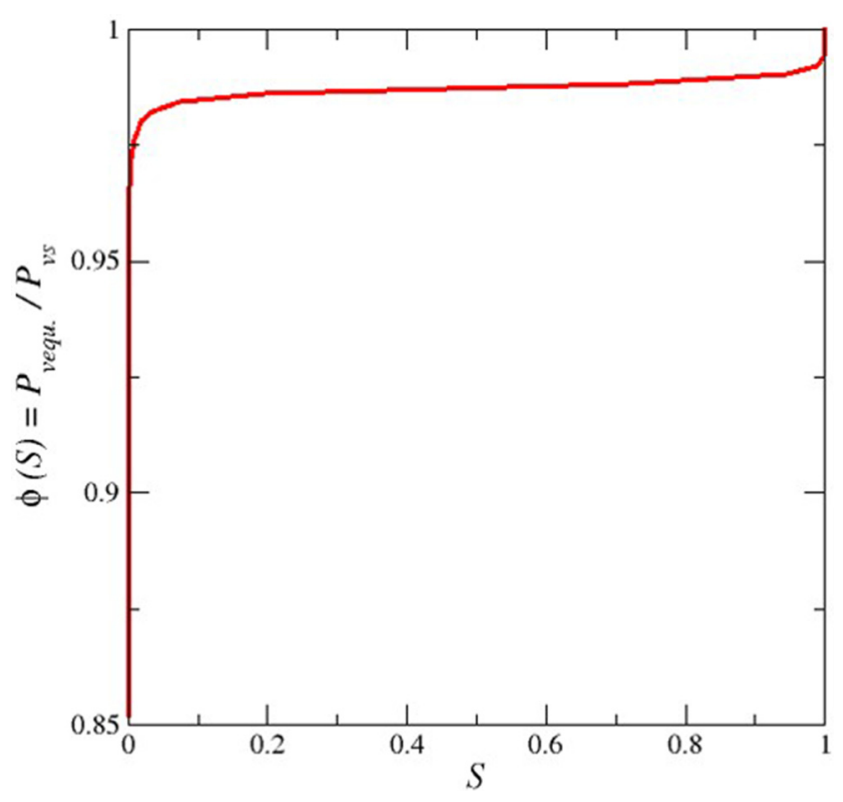

FIG. 11. Simulated desorption isotherm with the drying model and with the desorption isotherm algorithm (see the text). Both results are superimposed.

As expected, using this algorithm exactly leads to the same desorption curve (depicted in Fig. 11) as the one obtained from the drying simulation. This illustrates the fact that using a static drying model according to the classification mentioned in the introduction can be relevant. However, as illustrated later, a dynamic model is necessary to describe the transient evolution between two equilibrium situations.

\section{F. Non-local equilibrium effect}

As discussed, for instance, in Ref. 40, the classical continuum models of drying (see, e.g., Ref. 41) are based on the local equilibrium assumption. This means that the vapor pressure within a representative elementary volume (REV) is very close to the equilibrium vapor pressure. As a consequence, at the scale of the REV in which the average vapor pressure is denoted by $\left\langle P_{v}\right\rangle^{g}$, the equilibrium relationship to be considered is the desorption isotherm

$$
\left\langle P_{v}\right\rangle^{g}=P_{\text {vequ. }}=P_{v s} \phi(S) .
$$

This assumption has been questioned (see, for instance, ${ }^{11}$ ), and it is, therefore, of interest to get some insights on this question from our drying PNM simulations, including Kelvin effect. It must be emphasized that the non-local equilibrium discussed here has nothing to do with the non-equilibrium effect at an evaporating meniscus mentioned at the end of Sec. II in relation with Schrage's theory. ${ }^{25}$ In our model, the local thermodynamic equilibrium conditions are imposed at the menisci and the non-equilibrium effect (discussed here occurs at a different scale, namely the REV scale). The NLE considered here is due to the vapor concentration gradients induced by the drying process within the REV. To explore the REV non-local equilibrium effect, a 
procedure similar to the one described in Ref. 40 is followed. The average vapor pressure is determined within a slice as

$$
\left\langle P_{v}\right\rangle^{g}=\frac{\sum_{1}^{n_{g-s i c e}} \frac{\pi}{6} d_{p}^{3} P_{v}+\sum_{1}^{n_{p I-\text { slice }}} \frac{\pi}{6} d_{p}^{3}\left(1-S_{p}\right) P_{v}}{\sum_{1}^{n_{\text {slice }}} \frac{\pi}{6} d_{p}^{3}} .
$$

Similarly, the slice average saturation, $S_{s l .}$, is computed as

$$
S_{\text {sl. }}=\frac{\sum_{1}^{n_{\ell-\text { slice }}} \frac{\pi}{6} d_{p}^{3}+\sum_{1}^{n_{P I-\text { slice }}} \frac{\pi}{6} d_{p}^{3} S_{p}}{\sum_{1}^{n_{\text {slice }}} \frac{\pi}{6} d_{p}^{3}}
$$

In these two equations, $n_{g-\text { slice }}, n_{P I-s l i c e}$, and $n_{\ell-\text { slice }}$, respectively, denote the number of G, PI, and L pore bodies in the slice, while $n_{\text {slice }}$ represents the total number of pore bodies in the slice. Note that for simplicity, Eqs. (17) and (18) are written while assuming volumeless throats. When volumes are assigned to both throats and pore bodies, similar expressions are obtained.

The purpose is, hence, to analyze the average vapor pressure profiles together with the saturation profiles within the network (for $0 \leq z / H \leq 1$ ) for various overall saturations, $S$, i.e., at various times. For practical purposes, the slice average vapor pressure is normalized by the average equilibrium vapor pressure at all the clusters boundaries given by $P_{k}=\left\langle P_{c}\right\rangle=\frac{1}{N} \sum_{1}^{N} P_{c}$, where $P_{c}$ refers to a

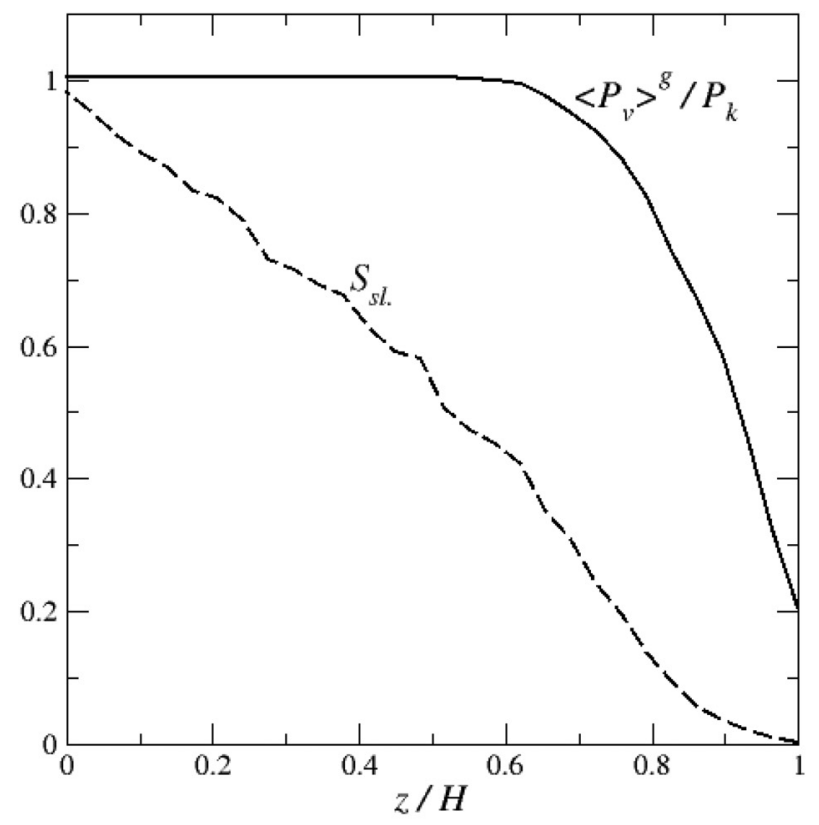

(a) cluster equilibrium vapor pressure and $N$ being the number of clusters. As in Ref. 40, and as illustrated in Fig. 2, a slice is understood as a domain containing one horizontal line of pore bodies, all the horizontal throats, and half the vertical throats adjacent to the pore bodies in the line.

Profiles resulting from this procedure for two values of the overall saturation, $S$, are reported in Fig. 12. They were obtained after averaging results obtained over 25 realizations of a $30 \times 30$ square network and a relatively thin external boundary layer, namely, $\delta=10 \times a$. Other conditions are the nominal conditions, namely, pore body and throat radii in the range $(65-135 \mathrm{~nm})$ and $(2-48 \mathrm{~nm})$, respectively, using uniform p.d.f. The external relative humidity $R H_{\infty}$ is equal to zero.

As can be observed from Fig. 12, the slice averaged vapor partial pressure is less than the slice averaged mean equilibrium vapor pressure, $P_{k}$, in slices, where the slice averaged saturation, $S_{s l}$, is non zero. More specifically, the difference between the two pressures becomes significant when $S_{s l}$ decreases below a threshold value (typically, this difference approximately exceeds $10 \%$ when $S_{\text {sl. }} \leq 0.2$ for $\mathrm{S}=0.5$ [Fig. $12(\mathrm{a})]$ and $S_{\text {sl. }} \leq 0.4$ for $\mathrm{S}=0.8$ [Fig. 12(b)]). This is an indication of a non-local equilibrium (NLE) effect.

To further illustrate this effect, an NLE function, $\phi_{N L E}=\left\langle P_{v}\right\rangle^{g} / P_{v s}$, may be defined which variation with the slice average saturation, $S_{s l}$, is represented in Fig. 13 for four values of the overall network saturation. In this figure, the desorption isotherm, $\phi(S)$ (see Fig. 11), is also reported for comparison purposes.

As can be seen, the NLE function is significantly different from the desorption isotherm, confirming a noticeable NLE effect. This is in agreement with the discussions in Ref. 11, claiming that NLE effects

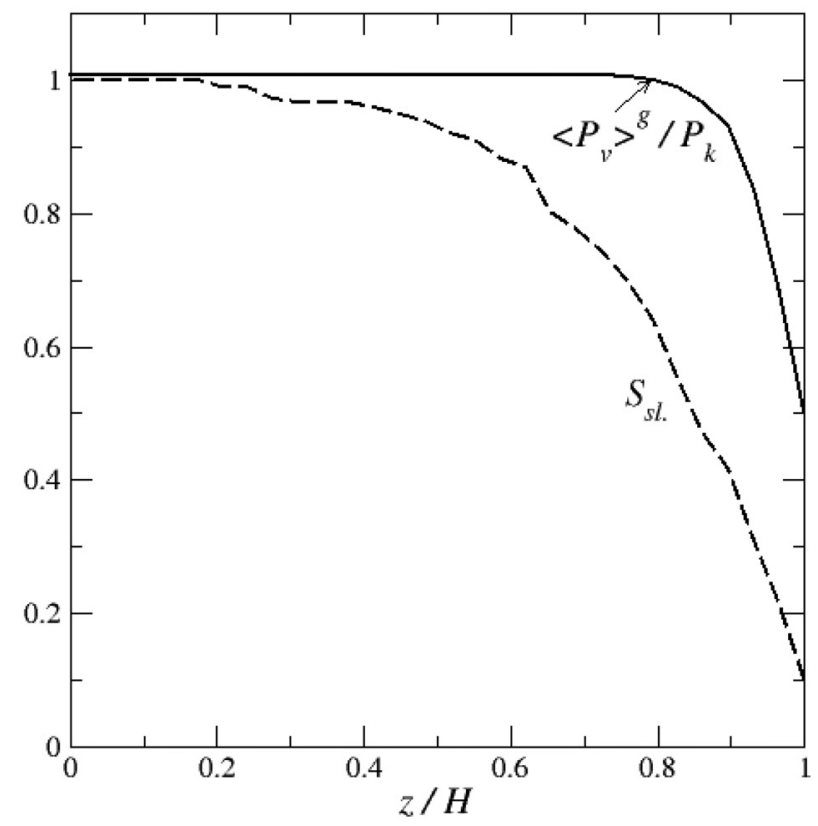

(b)

FIG. 12. Profiles of the slice-averaged normalized vapor partial pressure, $\frac{\left\langle P_{P}\right\rangle^{g}}{P_{k}}$, and the local saturation, $S_{s l}$, vs the normalized network height, $z / H$, obtained from the drying PNM at network overall saturations $S=0.5$ (a) and $S=0.8$ (b). Both quantities are resulting from an average over 25 realizations of a $30 \times 30$ network. 
must be considered in the modeling of drying within the framework of the continuum approach in porous media. It can also be seen that the NLE function varies with the overall network saturation. The variations with $S$ are qualitatively similar to the results reported in Ref. 40 in the absence of Kelvin effect. Even if it may be argued that the network under consideration is quite small and that a slice was not proven to be an acceptable REV, results exemplified in Fig. 13 are an additional indication in favor of the consideration of NLE effects in drying. Certainly, it would be interesting to perform similar computations over much larger networks and preferentially with 3D networks.

\section{G. Drying for $\delta \gg \mathbf{H}$}

As a final investigation, it is interesting to analyze the timeevolution of the evaporation rate for various values of $\delta \gg H$. At each time step, the vapor pressure is governed by the menisci curvature in the network. Thus, in this limit, one can assume that

$$
P_{v I}=P_{v s} \phi(S),
$$

where $P_{v I}$ denotes the vapor pressure at the interface and $\phi(S)$ is the desorption isotherm (see Fig. 11). The evaporation rate would then be given by

$$
E=A D_{v} \frac{M_{v}}{R T} \frac{\left(P_{v I}-P_{v \infty}\right)}{\delta}=A D_{v} \frac{M_{v}}{R T} P_{v s} \frac{\left(\phi(S)-R H_{\infty}\right)}{\delta},
$$

in which $A$ is the top surface area of the porous medium. Using the evaporation rate at $t=0$, given by $E_{0}=A D_{v} \frac{M_{v}}{R T} P_{v s} \frac{\left(1-R H_{\infty}\right)}{\delta}$, as the

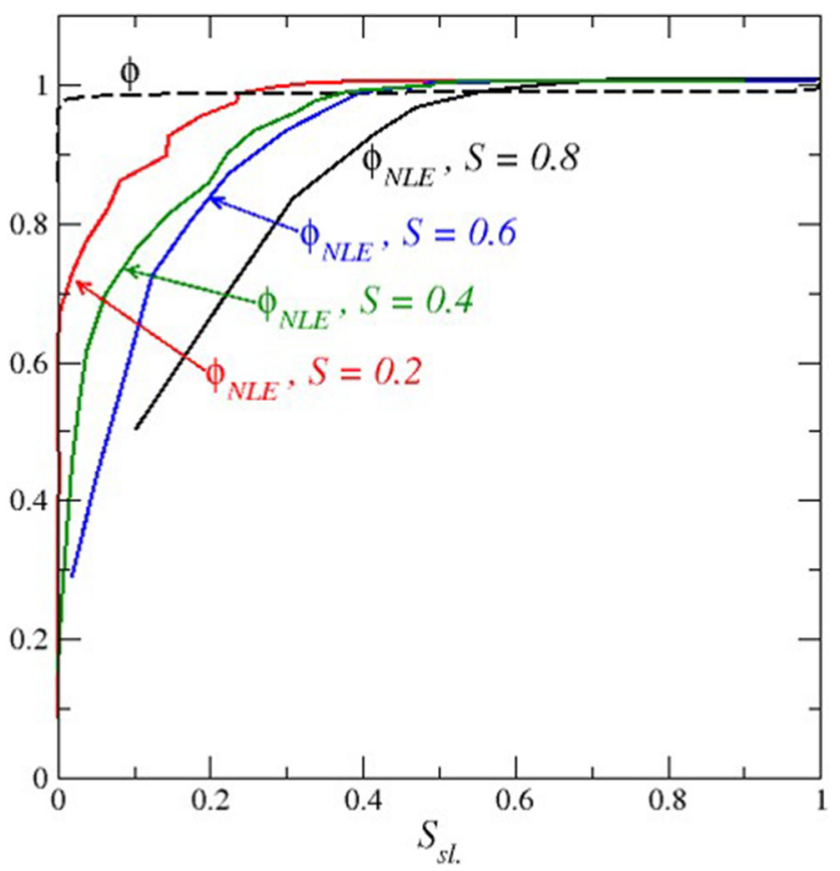

FIG. 13. Internal NLE function $\left(\phi_{N L E}\right)$ obtained from the PN drying simulations as a function of local saturation, $S_{s l}$, for different network overall saturation, $S$. The desorption isotherm is the curve labeled " $\phi$." reference, the normalized evaporation rate can be expressed, from Eq. (20), as

$$
\frac{E}{E_{0}}=\frac{\left(\phi(S)-R H_{\infty}\right)}{\left(1-R H_{\infty}\right)} .
$$

Results on $\frac{E}{E_{0}}$ obtained for a $30 \times 30$ square network are reported in Fig. 14 vs the network saturation, $S$. Here again, pore body diameters are in the range $(65-135 \mathrm{~nm})$, while throat diameters vary from 2 to $48 \mathrm{~nm}$, both following uniform p.d.f. The external relative humidity $R H_{\infty}$ is taken equal to zero, which, from Eq. (21), simply leads to $\frac{E}{E_{0}}=\phi(S)$. Three values of the diffusive layer thickness are considered: $\delta=10 \times a, \delta=1000 \times a$, and $\delta=10000 \times a$.

As can be seen from Fig. 14, the drying curve tends toward the curve obtained from the desorption isotherm [Eq. (21)] when the external diffusive layer thickness $\delta$ is increased to very large values compared to $H$. This is expected on the ground that the diffusive transfer resistance within the network must gradually become negligible compared to the external transfer resistance when $\delta$ is increased. However, it can be seen that the convergence is quite slow. For the largest value under investigation, which corresponds to a ratio $\frac{H}{\delta}$ as low as $3 \times 10^{-3}$, evaporation rates are still noticeably different. This may be related to the impact of the NLE effect previously discussed, indicating again that the mean vapor pressure in the network is different from the equilibrium vapor pressure.

\section{CONCLUSIONS}

A pore network model of isothermal drying, taking into account the Kelvin effect, was presented. This effect is noticeable for porous media with pore sizes in the mesoscopic range, typically with sizes

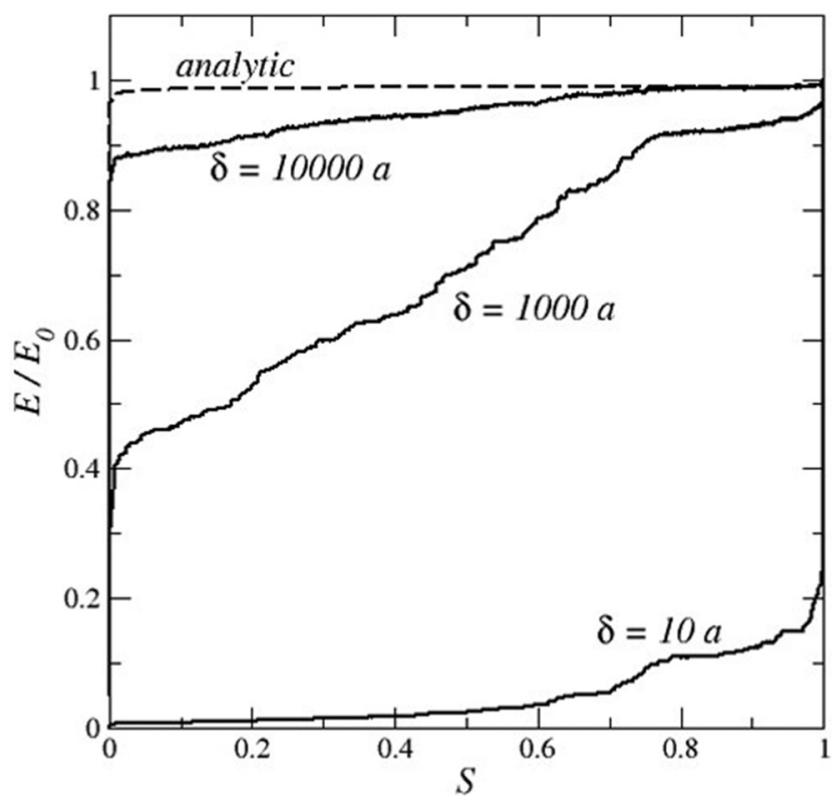

FIG. 14. Impact of the external diffusive layer thickness, $\delta$, on the drying curve. The curve labeled "analytic" corresponds to Eq. (21) [or, since $R H_{\infty}=0$, to $\phi(S)$ ]. 
varying between a few nanometers and $50 \mathrm{~nm}$. The model also takes into account the rarefaction effects on the vapor diffusion process with the consideration of Knudsen diffusion together with the usual molecular diffusion.

The drying PNM algorithm with Kelvin effect is noticeably more complicated than the drying PNM algorithm for capillary porous media for which the Kelvin effect can be ignored. The main point is to determine the curvature of the menisci at the boundary of the liquid clusters forming during the drying process. In the quasi-static limit considered in this work, the meniscus curvature is uniform at the boundary of a given cluster but can vary from one cluster to the other. The meniscus curvature for each cluster is determined through an iterative procedure using Kelvin relationship, so that the net mass transfer rate from each liquid cluster in the network is zero (evaporation at some regions of the cluster boundary is exactly balanced by condensation in the complementary regions of the cluster boundary) or greater than zero (due to net evaporation) at each step of the drying process.

Simulations were performed so as to illustrate the impact of the Kelvin effect. It was shown that this effect has an impact on the drying pattern, i.e., the liquid distribution in the network, compared to the situation where the Kelvin effect is neglected. The liquid distribution tends to be spatially more uniform compared to the situation with negligible Kelvin effect, with the presence of many isolated small cluster for which evaporation is not possible.

Depending on the pore size distribution (more exactly, the throat size distribution) and the relative humidity in the external gas phase, three main situations can be distinguished: no drying, partial drying, and full drying. Partial drying is obtained when the relative humidity in the gas phase external to the medium is smaller than the equilibrium vapor pressure corresponding to the largest throats in the network but larger than the equilibrium vapor pressure corresponding to the smallest throats in the network. Full drying is obtained when the relative humidity in the external gas phase is smaller than the equilibrium vapor pressure corresponding to the smallest throats in the network, whereas no drying occurs when the relative humidity in the external gas phase is larger than the equilibrium vapor pressure corresponding to the largest throats in the network.

When drying is partial, the saturation is non-zero in the sample at the end of drying process. An interesting question is whether this saturation is directly given by the desorption isotherm. Our simulations indicate that this is, indeed, the case in the purely capillary limit considered in this article. In the presence of significant viscous effects, it is expected that the final saturation be different from the one given by the desorption isotherm. More precisely, it is expected that the greater the viscous effects, the smaller the final saturation since viscous effects must allow invading throats that cannot be invaded when capillary effects are fully dominant. In this respect, it would be interesting to extent the present model so as to take into account the viscous effects by first adapting the algorithm developed in the absence of Kelvin effect (see, e.g., Ref. 42).

Simulations performed in this work indicate that the evaporation rate fluctuates with possible local (in time) slight re-increase as a result of menisci curvature variations during the drying process. This feature is directly related to the Kelvin effect and not observed in PNM simulations neglecting the Kelvin effect. One might argue that the simulations reported in the present analysis were performed on networks of relatively small size. The question may then arise as to whether fluctuations will be damped out in larger networks. Exploring this question is left for a future work.

Simulations reported here also indicate a noticeable NLE effect. This certainly needs to be confirmed by simulations over much larger networks so as to consider presumably more adequate averaging volumes than the horizontal thin slices considered in the present work (a somewhat standard view is to consider that an REV is a cubic or spherical domain of sizes corresponding to at least 10-20 lattice spacing in each direction). Nevertheless, our results strongly support the idea that NLE models are more adapted to describe the drying process than the much more classical local equilibrium models, not only in the case of the capillary porous media ${ }^{40}$ but also for hygroscopic porous materials.

Naturally, the present model relies on several simplifying assumptions, which deserve to be revisited in future works. In addition to the already mentioned viscous effects, these include the possible effect of cavitation, ${ }^{13,17}$ as well as the possible impact of the total gas pressure. ${ }^{10}$ The latter effect is somewhat controversial. ${ }^{43}$ In this regard, PNM simulations could help settle the question, at least in the case of isothermal drying. Also, the flow in adsorbed and capillary liquid films (see, e.g., Refs. 6 and 16) has been neglected. For this reason, the model presented in this article is only seen as a first step in the development of PNM for the study of drying in mesoporous materials. However, it is surmised that the key aspect addressed in this article, i.e., the computation of the menisci curvature via the Kelvin effect at the boundary of each liquid cluster, will also be a key aspect in the presence of significant film effects.

\section{ACKNOWLEDGMENTS}

Financial support from joint project "Drycap," funded by GIP ANR (Project No. 16-CE92-0030-01) and DFG (Project No. TS28/ $10-1)$, is gratefully acknowledged.

\section{DATA AVAILABILITY}

The data that support the findings of this study are available from the corresponding author upon reasonable request.

\section{REFERENCES}

'Z. P. Bažant and L. J. Najjar, "Drying of concrete as nonlinear diffusion problem,” Cem. Concr. Res. 1(5), 461 (1971).

${ }^{2}$ T. Humplik, J. Lee, S. O’Hern, B. Fellman, M. Baig, S. Hassan, M. Atieh, F. Rahman, T. Laoui, and R. Karnik, "Nanostructured materials for water desalination," Nanotechnology 22, 292001 (2011).

${ }^{3}$ D. Coso, V. Srinivasan, M. C. Lu, J. Y. Chang, and A. Majumdar, "Enhanced heat transfer in biporous wicks in the thin liquid film evaporation and boiling regimes," J. Heat Transfer 134, 101501 (2012).

${ }^{4}$ R. H. Rand, "Fluid mechanics of green plants," Annu. Rev. Fluid Mech. 15, 29 (1983).

${ }^{5}$ A. W. Adamson, Physical Chemistry of Surfaces, 5th ed. (Wiley, 1990).

${ }^{6} \mathrm{P}$. Chen and D. C. T. Pei, "A mathematical model of drying process," Int. J. Heat Mass Transfer 32(2), 297 (1989).

${ }^{7}$ D. P. Bentz, E. J. Garboczi, and D. A. Quenard, "Modelling drying shrinkage in reconstructed porous materials: Application to porous Vycor glass," Modell. Simul. Mater. Sci. Eng. 6, 211 (1998).

${ }^{8}$ G. E. Tabbal, P. Dangla, M. Vandamme, M. Bottoni, and S. Granet, "Modelling the drying shrinkage of porous materials by considering both capillary and adsorption effects," J. Mech. Phys. Solids 142, 104016 (2020). 
${ }^{9}$ L. Pel, K. A. Landman, and E. F. Kaasschierter, "Analytic solution for the nonlinear drying problem," Int. J. Heat Mass Transfer 45(15), 3173 (2002).

${ }^{10} \mathrm{M}$. Mainguy, O. Coussy, and V. Baroghel-Bouny, "Role of air pressure in drying of weakly permeable materials," J. Eng. Mech. 127, 582 (2001).

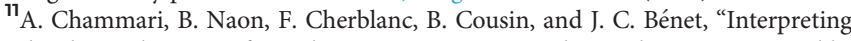
the drying kinetics of a soil using a macroscopic thermodynamic nonequilibrium of water between the liquid and vapor phase," Drying Technol. 26, 836 (2008).

${ }^{12}$ H. Penvern, M. Zhou, B. Maillet, D. Courtier-Murias, M. Scheel, J. Perrin, T. Weitkamp, S. Bardet, S. Carré, and P. Coussot, "How bound water regulates wood drying," Phys. Rev. Appl. 14(5), 054051 (2020).

${ }^{13}$ C. Duan, R. Karnik, M. C. Lu, and A. Majumdar, "Evaporation-induced cavitation in nanofluidic channels," Proc. Natl. Acad. U. S. A. 109(10), 3688 (2012).

${ }^{14}$ E. Keita, S. A. Koehler, P. Faure, D. A. Weitz, and P. Coussot, "Drying kinetics driven by the shape of the air/water interface in capillary channel," Eur. Phys. J. E39(2), 23 (2016).

${ }^{15} \mathrm{~F}$. Chauvet, P. Duru, S. Geoffroy, and M. Prat, "Three periods of drying of a single square capillary tube," Phys. Rev. Lett. 103, 124502 (2009).

${ }^{16}$ J. C. T. Eijkel, B. Dan, H. W. Reemeijer, D. C. Hermes, J. G. Bomer, and A. van den Berg, "Strongly accelerated and humidity independent drying of nanochannels induced by sharp corners," Phys. Rev. Lett. 95, 256107 (2005).

${ }^{17}$ O. Vincent, D. A. Sessoms, E. J. Huber, J. Guioth, and A. D. Stroock, "Drying by cavitation and poroelastic relaxations in porous media with macroscopic pores connected by nanoscale throats," Phys. Rev. Lett. 113(13), 134501 (2014).

${ }^{18}$ D. Panda, S. Paliwal, D. P. Sourya, A. Kharaghani, E. Tsotsas, and V. K. Surasani, "Influence of thermal gradients on the invasion patterns during drying of porous media: A lattice Boltzmann method," Phys. Fluids 32, 122116 (2020).

${ }^{19} \mathrm{M}$. Prat, "Percolation model of drying under isothermal conditions in porous media," Int. J. Multiphase Flow 19(4), 691 (1993).

${ }^{20} \mathrm{M}$. Prat, "Pore network models of drying, contact angle and films flows," Chem. Eng. Technol. 34(7), 1029 (2011).

${ }^{21} \mathrm{~T}$. Metzger, "A personal view on pore network models in drying technology," Drying Technol. 37, 497 (2019).

${ }^{22}$ T. Takei, M. Chikazawa, and T. Kanazawa, "Validity of the Kelvin equation in estimation of small pore size by nitrogen adsorption," Colloid Polym. Sci. 275, 1156 (1997).

${ }^{23}$ A. G. Yiotis, D. Salin, E. S. Tajerand, and Y. C. Yortsos, "Drying in porous media with gravity-stabilized fronts: Experimental results," Phys. Rev. E 86, 026310 (2012).

${ }^{24} \mathrm{M}$. Prat, "On the influence of pore shape, contact angle and film flows on drying of capillary porous media," Int. J. Heat Mass Transfer 50, 1455 (2007).

${ }^{25} \mathrm{R}$. W. Schrage, A Theoretical Study of Interphase Mass Transfer (Columbia University Press, 1953).

${ }^{26} \mathrm{Z}$. Lu, S. Narayanan, and E. N. Wang, "Modeling of evaporation from nanopores with nonequilibrium and nonlocal effects," Langmuir 31, 9817 (2015).
${ }^{27}$ V. Joekar-Niasar, S. M. Hassanizadeh, and H. Dahle, "Dynamic pore-network modeling of drainage in two-phase flow," J. Fluid Mech. 655, 38 (2010).

${ }^{28}$ T. Metzger, E. Tsotsas, and M. Prat, "Pore network models: A powerful tool to study drying at the pore level and understand the influence of structure on drying kinetics," Mod. Drying Technol. 1, 57-102 (2007).

${ }^{29}$ A. G. Yiotis, A. K. Stubos, A. G. Boudouvis, and Y. C. Yortsos, "A 2D porenetwork model of the drying of single-component liquids in porous media," Adv. Water Resources 24, 439 (2001).

${ }^{30} \mathrm{D}$. Wilkinson and J. F. Willemsen, "Invasion percolation: A new form of percolation theory," J. Phys. A 16, 3365 (1983).

${ }^{31}$ J. H. Page, J. Liu, B. Abeles, E. Herbolzheimer, H. W. Deckman, and D. A. Weitz, "Adsorption and desorption of a wetting fluid in Vycor studied by acoustic and optical techniques," Phys; Rev. E 52(3), 2763 (1995).

${ }^{32}$ A. Naillon, H. Massadi, R. Courson, L. Seveno, P. F. Calmon, M. Prat, and P. Joseph, "Quasistatic drainage in nano-slits network with non-uniform depth designed by grayscale laser lithography," Microfluid. Nanofluid. 21(8), 131 (2017).

${ }^{33}$ F. Babalievski, "Cluster counting: The Hoshen-Kopelman algorithm versus spanning tree approaches," Int. J. Mod. Phys. C 9(1), 43 (1998).

${ }^{34}$ C. H. Bosanquet, British TA Report BR-507 (1944).

${ }^{35}$ P. J. A. M. Kerkhof and M. A. M. Geboers, "Toward a unified theory of isotropic molecular transport phenomena," AIChE J. 51, 79 (2005).

${ }^{36}$ W. B. Krantz, A. R. Greenberg, E. Kujundzic, A. Yeo, and S. S. Hosseini, "Evapoporometry: A novel technique for determining the pore-size distribution of membranes," J. Membr. Sci. 438, 153 (2013).

${ }^{37} \mathrm{O}$. Chapuis and M. Prat, "Influence of wettability conditions on slow evaporation in two-dimensional porous media," Phys. Rev. E 75, 046311 (2007).

${ }^{38} \mathrm{H}$. Chraibi, M. Prat, and O. Chapuis, "Influence of contact angle on slow evaporation in two dimensional porous media," Phys. Rev. E 79, 026313 (2009).

${ }^{39}$ M. E. Hannach, T. Soboleva, K. Malek, A. A. Franco, M. Prat, J. Pauchet, and S. Holdcroft, "Characterization of pore network structure in catalyst layers of polymer electrolyte fuel cells," J. Power Sources 247, 322 (2014).

${ }^{40}$ A. A. Moghaddam, M. Prat, E. Tsotsas, and A. Kharaghani, "Evaporation in capillary porous media at the perfect piston-like invasion limit: Evidence of non-local equilibrium effects," Water Resources Res. 53(12), 10433 (2017).

${ }^{41} \mathrm{~S}$. Whitaker, "Simultaneous heat, mass, and momentum transfer in porous media: A theory of drying," Adv. Heat Transfer 13, 119 (1977).

${ }^{42} \mathrm{~T}$. Metzger, A. Irawan, and E. Tsotsas, "Isothermal drying of pore networks: Influence of friction for different pore structures," Drying Technol. 25, 49 (2007).

${ }^{43}$ M. Thiery, V. Baroghel-Bouny, N. Bourneton, G. Villain, and C. Stéfani, "Modélisation du séchage des bétons, analyse des différents modes de transfert hydrique," Rev. Eur. Gén. Civil 11(5), 541 (2007). 